\title{
Phylogenetic and syntenic data support a single horizontal transference to a Trypanosoma ancestor of a prokaryotic proline racemase implicated in parasite evasion from host defences
}

\author{
Zuleima C Caballero ${ }^{1,2+}$, Andre G Costa-Martins ${ }^{1 \dagger}$, Robson C Ferreira ${ }^{1}$, João M P Alves ${ }^{1}$, Myrna G Serrano ${ }^{3}$, \\ Erney P Camargo ${ }^{1}$, Gregory A Buck ${ }^{3}$, Paola Minoprio ${ }^{4}$ and Marta M G Teixeira ${ }^{1 *}$
}

\begin{abstract}
Background: Proline racemase (PRAC) enzymes of Trypanosoma cruzi (TCPRAC), the agent of Chagas disease, and Trypanosoma vivax (TVPRAC), the agent of livestock trypanosomosis, have been implicated in the B-cells polyclonal activation contributing to immunosuppression and the evasion of host defences. The similarity to prokaryotic PRAC and the absence in Trypanosoma brucei and Trypanosoma congolense have raised many questions about the origin, evolution, and functions of trypanosome PRAC (TryPRAC) enzymes.

Findings: We identified TryPRAC homologs as single copy genes per haploid genome in 12 of 15 Trypanosoma species, including T. cruzi and T. cruzi marinkellei, T. dionisii, T. erneyi, T. rangeli, T. conorhini and T. lewisi, all parasites of mammals. Polymorphisms in TCPRAC genes matched T. cruzi genotypes: TCl-TcIV and Tcbat have unique genes, while the hybrids TCV and TCVI contain TCPRACA and TCPRACB from parental TCll and TcIll, respectively. PRAC homologs were identified in trypanosomes from anurans, snakes, crocodiles, lizards, and birds. Most trypanosomes have intact PRAC genes. T. rangeli possesses only pseudogenes, maybe in the process of being lost. T. brucei, T. congolense and their allied species, except the more distantly related T. vivax, have completely lost PRAC genes.

Conclusions: The genealogy of TryPRAC homologs supports an evolutionary history congruent with the Trypanosoma phylogeny. This finding, together with the synteny of PRAC loci, the relationships with prokaryotic PRAC inferred by taxon-rich phylogenetic analysis, and the absence in trypanosomatids of any other genera or in bodonids or euglenids suggest that a common ancestor of Trypanosoma gained PRAC gene by a single and ancient horizontal gene transfer (HGT) from a Firmicutes bacterium more closely related to Gemella and other species of Bacilli than to Clostridium as previously suggested. Our broad phylogenetic study allowed investigation of TryPRAC evolution over long and short timescales. TryPRAC genes diverged to become species-specific and genotype-specific for T. cruzi and T. rangeli, with resulting genealogies congruent with those obtained using vertically inherited genes. The inventory of TryPRAC genes described here is the first step toward the understanding of the roles of PRAC enzymes in trypanosomes differing in life cycles, virulence, and infection and immune evasion strategies.
\end{abstract}

Keywords: Proline racemase, Trypanosoma cruzi, Trypanosoma vivax, Trypanosoma rangeli, Horizontal gene transfer, Gene loss, Kinetoplastid evolution, Phylogeny, Synteny, Genotyping

\footnotetext{
* Correspondence: mmgteix@icb.usp.br

'Equal contributors

'Departamento de Parasitologia, Instituto de Ciências Biomédicas,

Universidade de São Paulo, São Paulo, SP 05508-900, Brazil

Full list of author information is available at the end of the article
} 


\section{Background}

The kinetoplastids (Euglenozoa: Kinetoplastea) are composed of bodonids, which include free-living and parasitic species in aquatic environments, and their descendants, the obligate parasitic trypanosomatids. These include parasites of insects and plants as well as Trypanosoma and Leishmania, which alternate between invertebrate and vertebrate hosts, including humans [1-3]. Although the trypanosomes are parasites of all vertebrate classes, they are highly divergent in their host ranges. Some species have a single vertebrate host, while others can infect diverse genera and orders. Most trypanosomes are thought to develop exclusively in the bloodstream, but a few species can also live in extra-vascular (T. brucei ssp. and T. vivax) and intracellular (T. cruzi and allied species) compartments. In vectors such as flies, hemipterans, mosquitoes, fleas or leeches, most trypanosomes develop in the gut, although some can invade the haemolymph and multiply and differentiate to infective forms in the salivary glands of their vectors [1,3-5].

Morphological and functional diversification has given rise to trypanosomatids differing in life cycles, vertebrate hosts, and vectors. Parasite adaptations to the variable host and vector environments have resulted in the development of diverse physiological processes and unique mechanisms to evade the host defences. Characterisation of molecules essential to metabolism and host interactions is fundamental to the elucidation of the emergence of pathogenicity and the diverse evolutionary strategies used by trypanosomes to infect and survive within a wide range of vertebrates and invertebrates.

Polyclonal lymphocyte-B activation is one of the major immunological disorders observed during microbial infections and is among the main strategies used by $T$. cruzi to evade the host specific immune response, ensuring its survival in vertebrate hosts [6-11]. This process can be triggered by proline racemase (PRAC) enzymes released by $T$. cruzi, which are implicated in the virulence of the parasite and induce indiscriminate activation of B-cells producing high levels of non-specific antibodies that contribute to immunosuppression and, consequently, to parasite immune evasion and persistence in the host. Immunological and biochemical studies confirmed that both TcPRAC (T. cruzi) and TvPRAC ( $T$. vivax) exhibited proline racemase activity and B-cell mitogenicity, inducing polyclonal activation, delayed specific immune responses to parasite antigens favouring parasite immune evasion, and concomitant increase of parasitemias in the early phase of infection [7-12].

Amino-acid racemases are enzymes that catalyse the interconversion of free $\mathrm{L}$ - and $\mathrm{D}$-amino acids. D-amino acids released by bacteria are key factors for the cell wall remodelling essential for adaptation to environmental challenges. Alanine- and glutamate-racemases are necessary for the synthesis of bacterial cell wall (peptidoglycan), which provide protection against proteolysis and host immune defences [13-15]. The enzymatic activity of Proline-racemase (PRAC) enzymes has been identified in a restricted group of bacteria, acting as a virulence factor in the highly pathogenic Clostridium difficile and Pseudomonas aeruginosa [14-16]. The first PRAC enzyme was isolated from Clostridium sticklandii in 1968 [15]. The first eukaryotic PRAC was reported in 2000 in T. cruzi [11]. In 2009, a PRAC was reported in T. vivax [8]. It is now recognised that the PRAC-like gene family is widely distributed throughout prokaryotes but scarce in eukaryotes, which according to phylogenetic analyses have acquired distinct bacterial PRAC-like genes by independent horizontal gene transfer (HGT) events. The repertoires and roles of PRAC-like genes in eukaryotes other than trypanosomes, including fungi, humans and other animals, are just beginning to be appreciated $[16,17]$.

The search for PRAC genes in the genome of the $T$. cruzi CL Brener strain revealed two genes encoding two enzyme isoforms essential for viability and differentially expressed during parasite development: TcPRACA (secreted by metacyclic and bloodstream trypomastigotes) and TcPRACB (intracellular protein of epimastigotes). The two enzymes share $96 \%$ amino-acid identity but differ in kinetic properties relevant to catalytic activities $[9,10]$. Evidence provided by inhibitors of TcPRAC support its suitability as a target for chemotherapy against Chagas disease $[18,19]$. However, to date neither the genetic nor the enzymatic diversity of TcPRAC was investigated for any other strain of $T$. cruzi besides CL Brener. All protein candidates for drug design should consider the diversity within T. cruzi [20].

$T$. cruzi is a complex of genetically diverse isolates distributed in seven intraspecific subdivisions: the DTUs (Discrete Typing Units) TcI-TcVI and Tcbat. The heterogeneity of T. cruzi isolates has been implicated in different clinical forms of the disease. Chagas disease pathology ranges from subclinical infection to severe cardiac and digestive syndromes. However, attempts to associate T. cruzi genotypes with clinical forms, degrees and types of host-cell invaded, virulence and metacyclogenesis suggested some degrees of association, but involved several factors from hosts and parasites that are not well understood [21].

As mentioned above, TcPRAC enzymes contribute to delays in the effective host immune response by nonspecifically activating B-lymphocytes, thus enhancing the ability of the parasite to avoid immune clearance $[8,9,11]$. Treatment of macrophages with recombinant TcPRAC induces the secretion of a soluble factor that promotes B-cell proliferation. TcPRAC also activates the production of a cytokine known to enhance host susceptibility to $T$. cruzi, thus enhancing parasite virulence $[22,23]$. Over-expression of TcPRAC genes increased the 
differentiation of non-infective epimastigotes into infective metacyclic trypomastigotes, suggesting that the enzyme may regulate intracellular metabolic pathways of L-proline internalised from the vector gut. The inhibition of TcPRAC significantly reduced the invasion of cells, and the intracellular differentiation of $T$. cruzi $[9,10]$. L-proline is one major source of energy for $T$. cruzi not only in the vectors but also during host-cell invasion and, in addition, improves parasite protection against oxidative stress [24,25]. TcPRAC may also participate in the addition of D-amino acids to peptides, generating less immunogenic parasites, and maybe providing resistance against host proteolytic mechanisms as described for bacterial cell walls [10,12-14,16,22].

To date, the only trypanosome other than T. cruzi in which a PRAC homolog was reported is T. vivax. Similar to TcPRAC, TvPRAC displays racemase enzymatic activity, and induces polyclonal activation (mitogenic activity) in B-cells [8]. T. vivax evades the host immune system due to VSG expression, multiplying extracellularly in the bloodstream, and invading and multiplying in tissue spaces and the CNS similarly to the T. brucei ssp. agents of Sleeping Sickness [26,27].

Throughout their evolutionary history, trypanosomes have relied on various strategies to infect their hosts, obtain energy from sources available in vectors (gut and haemolymph) and vertebrate hosts (blood, intravascular and intracellular compartments), evade host defences, and develop virulence factors that play different roles according to the trypanosome species. PRAC enzymes have been implicated in these processes in T. cruzi and T. vivax [7-12]. The discovery of PRAC enzymes in $T$. cruzi and $T$. vivax, which are species separated by large genetic distances, and the absence of homologs in the genomes of T. brucei and T. congolense, which together with $T$. vivax form the clade T. brucei exclusive of African pathogenic trypanosomes $[3,28]$ and the lack in Leishmania spp. [8], suggest that PRAC have a complex evolutionary history in the Trypanosomatidae family.

The acquisition by HGT of a large number of foreign genes from viruses, bacteria, eukaryotes and even vertebrate hosts and vectors can change genetic and metabolic repertoires, and has played important roles in the evolution of trypanosomatids and other protistan parasites. HGT has been an important evolutionary force in the adaptation of trypanosomatids to parasitism and to specialised niches within hosts, largely contributing to amino acid and carbohydrate metabolic pathways. In addition, an increasing number of putative proteins of unknown function gained from bacterium have been identified in the T. brucei, T. cruzi and Leishmania spp. genomes [29-35]. Recent studies have characterised putative HGT contributing to host infection, cell invasion, virulence, and pathogenesis of trypanosomatids. It was suggested that $T$. cruzi acquired genes for the calcium mobilisation necessary for host-cell invasion via ancient HGT from Salmonella [36]. The analysis of the phosphatidylinositol kinase gene family revealed a novel gene of T. cruzi, T. brucei, T. congolense, T. vivax, and Leishmania spp. that may have been acquired from a virus through HGT [37].

To achieve a better understanding of the origin, the possible bacterial donors and the evolution of TryPRAC genes, we searched for TcPRAC homologs in the genomes of $T$. cruzi representing the whole range of intra-specific diversity (DTUs TcI-TcVI and Tcbat), other trypanosomes of mammals (T. c. marinkellei, T. dionisii, T. erneyi, T. rangeli, T. conorhini and T. lewisi), trypanosomes of snakes, crocodiles, toads, lizards and birds, trypanosomatids of several other genera, and bodonids and euglenids. Here, we describe PRAC repertoires of species and genotypes of trypanosomes, taxon-rich phylogeny of eukaryotic and prokaryotic PRAC homologs, GC contents, selection pressures on the evolution of TryPRAC, and genome synteny analyses. Together, the results allowed us to hypothesise about the origin, and number and timing of PRAC transference that gave rise to TryPRAC genes.

\section{Methods}

\section{Trypanosome genomes used for searches of TCPRAC} homologous genes

Searches for TcPRAC homologs were performed by BLAST against draft and annotated genomes of trypanosomatids freely available in TriTrypDB, geneDB and NCBI data banks. Sequences of TcPRAC and TvPRAC [10] were used as queries; full-length sequences and specific motifs from PRAC-like gene family were used as baits for the genome analyses of T. cruzi CL Brener, Silvio X10 plus other strains of T. cruzi (Table 1) sequenced by the Kinetoplastid Genome Sequencing and Analysis Consortium NIH/NHGRI/NIAID, T. brucei ssp., T. evansi, $T$. congolense, T. vivax, T. c. marinkellei, T. grayi, and species of Leishmania (Table 1). In addition, we examined the freely available genomes from Crithidia acanthocephali, Angomonas desouzai, Angomonas deanei, Strigomonas culicis, Strigomonas oncopelti and Herpetomonas muscarum, all generated in our laboratories, plus genomes from Phytomonas sp., Crithidia fasciculata and Endotrypanum schaudinni (Table 1).

We also searched for PRAC genes in genomes that have been generated in our laboratories for a large number of euglenozoans within the ATOL (Assembling the Tree of Life, NSF-USA) and TCC-USP (Brazil) projects aiming highly comprehensive phylogenomic inferences. The following ongoing genomes were analyzed: T. cruzi (G and Tcbat), T. cruzi marinkellei (TCC344), T. dionisii (TCC211), T. erneyi (TCC1946), T. rangeli (AM80), T. lewisi (TCC34), T. conorhini (TCC025E), T. serpentis 
Table 1 Trypanosomes, other trypanosomatids and free living kinetoplastids and euglenids examined in this study, and respective sequences of TCPRAC homologous genes

\begin{tabular}{|c|c|c|c|}
\hline Species isolate (genotype) & Host species & Data bank assessed & $\begin{array}{l}\text { TryPRACAccess nu } \\
\text { Genome/GenBank }\end{array}$ \\
\hline \multicolumn{4}{|l|}{ Trypanosomes } \\
\hline \multicolumn{4}{|l|}{ Trypanosoma cruzi } \\
\hline Sylvio X10.6 (Tcl) & Homo sapiens & TritrypDB \& & TCSYLVIO_010607 \\
\hline $\mathrm{JR}$ Cl4 (TCl) & Homo sapiens & Genome draft (WU) \& & KP001304 \\
\hline $\mathrm{G}(T \mathrm{Cl})$ & Didelphis marsupialis & Genome draft (ATOL) \# & KP001302 \\
\hline Esmeraldo (Tcll) & Homo sapiens & Genome draft (WU) \& & KP001301 \\
\hline M6241 cl6 (TcIII) & Homo sapiens & Genome draft (WU) \& & KP001305 \\
\hline Can III (TcIV) & Homo sapiens & Genome draft (WU) \& & KP001298 \\
\hline CLBrener -Esm (TcVI) & Triatoma infestans & TritrypDB \& & TCCLB.506795.80 \\
\hline CLBrener-Non-Esm (TcVI) & Triatoma infestans & TritrypDB \& & TCCLB.509935.29 \\
\hline Tula cl2 (TcVl) & Homo sapiens & Genome draft (WU) \& & KP001312 \\
\hline 1994 (Tcbat) & Myotis levis (bat) & Genome draft (USP) & KP001313 \\
\hline Trypanosoma cruzi marinkellei B7 & Phyllostomus discolor (bat) & TritrypDB \& & TC_MARK_8728 \\
\hline TCC344 & Carollia perspicillata (bat) & Genome draft (ATOL) \# & KP001314 \\
\hline Trypanosoma dionisii TCC211 & Eptesicus brasiliensis (bat) & Genome draft (ATOL) \# & KP001263 \\
\hline Trypanosoma erneyi TCC1946 & Mops condylurus (bat) & Genome draft (ATOL) \# & KP001315 \\
\hline Trypanosoma rangeli AM80 & Homo sapiens & Genome draft (ATOL) \# & KP001264 \\
\hline Trypanosoma conorhini TCC025 & Rattus rattus & Genome draft (ATOL) \# & KP001316 \\
\hline Trypanosoma lewisi TCC034 & Rattus rattus & Genome draft (ATOL) \# & KP001317 \\
\hline Trypanosoma vivax Y486 & Bos taurus & TritrypDB \& & TVY486_0703770 \\
\hline Trypanosoma b. brucei TREU927 & Glossina pallidipes & TritrypDB \& & \\
\hline Trypanosoma b. gambiense DAL972 & Homo sapiens & TritrypDB \& & \\
\hline Trypanosoma congolense IL3000 & Bos sp. & TritrypDB \& & \\
\hline Trypanosoma serpentis TCC1052 & Pseudoboa nigra (snake) & Genome draft (USP) \# & KP001318 \\
\hline Trypanosoma grayi ANR4 & Glossina palpalis & TritrypDB \& & Tgr.146.1080 \\
\hline Trypanosoma sp. TCC339 & Rhinella marina (toad) & Genome draft (USP) \# & KP001319 \\
\hline Trypanosoma sp. TCC1825 & Ramphocelus nigrogularis (bird) & Genome draft (USP) \# & KP001320 \\
\hline Trypanosoma sp. TCC878 & Mabuya frenata (lyzard) & Genome draft (USP) \# & KP001321 \\
\hline \multicolumn{4}{|l|}{ Other Trypanosomatids } \\
\hline Crithidia fasciculata & Anopheles quadrimaculatus & TritrypDB \& & \\
\hline Crithidia acanthocephali & Acanthocephala femorata (fly) & (ATOL) \& GenBank AUXI01000000 & \\
\hline Leptomonas costaricensis & Ricolla simillima (Hemiptera) & (ATOL) \# & \\
\hline Leishmania major Friedlin & Homo sapiens & TritrypDB \& & \\
\hline Leishmania tarentolae & Tarentola mauritanica (lyzard) & TritrypDB \& & \\
\hline Endotrypanum schaudinni & Choloepus hoffmani (sloth) & Genome draft (ATOL) \# & \\
\hline Angomonas desouzai & Ornidia obesa (fly) & (ATOL) \& GenBank AUXL01000000 & \\
\hline Angomonas deanei & Zelus leucogrammus (Hemiptera) & (ATOL) \& GenBank AUXM01000000 & \\
\hline Strigomonas culicis & Aedes vexans & (ATOL) \& GenBank AUXH01000000 & \\
\hline Strigomonas oncopelti & Oncopeltus sp. (Hemiptera) & (ATOL) \& GenBank AUXK01000000 & \\
\hline Herpetomonas muscarum & Musca domestica & (ATOL) \& GenBank AUXJ01000000 & \\
\hline Phytomonas sp. & Jatropha macrantha (plant) & Genome draft (ATOL) \# & \\
\hline
\end{tabular}


Table 1 Trypanosomes, other trypanosomatids and free living kinetoplastids and euglenids examined in this study, and respective sequences of TCPRAC homologous genes (Continued)

\begin{tabular}{ll}
\hline Free living euglenozoans & Genome draft (ATOL) \# \\
Bodo sp. ATCC 50149 & Genome draft (ATOL) \# \\
Parabodo caudatus ATCC 30905 & Genome draft (ATOL) \# \\
Discoplastis spatirhyncha SAG1224.42 & Genome draft (ATOL) \# \\
Eutreptia viridis SAG 1226-1C & \\
\hline TCC: Trypanosomatid Culture Collection of the University of São Paulo, SP, Brazil. & \\
TritrypDB (http://tritrypdb.org). & \\
WU: Washington University (USA) - Kinetoplastid Genome Sequencing and Analysis Consortium (NIH/NHGRI/NIAID). \\
ATOL: Assembling the Tree of Life (NSF-USA); \\
USP: Department of Parasitology, University of São Paulo, USP. \\
\& publicly available genomes; \# access to these ongoing genomes can be obtained by contacting the corresponding author.
\end{tabular}

(TCC1052), Trypanosoma sp. of toad (TCC339), Trypanosoma sp. of lizard (TCC878) and Trypanosoma sp. of bird (TCC1825). PRAC genes were also searched in draft genomes of bodonids (Bodo sp. and Parabodo caudatus), and euglenids (Euglena gracilis, Eutrepia viridis, Discoplastis spathirhyncha) (Table 1). The trypanosomatids employed for genome sequencing are cryopreserved at Trypanosomatid Culture Collection of the University of São Paulo (TCC-USP). PRAC sequences retrieved from the genomes were all deposited in GenBank (Table 1).

The draft genomes generated in our laboratories were sequenced using standard pyrosequencing shotgun methodology according to Roche 454 protocols and assembled by Roche's Newbler software (version 2.3) as previously described $[32,33]$. The ongoing genomes from trypanosomes of toad, snake, lizard and bird were obtained using the MiSeq Illumina plataform (mate-pair reads), and assembled using Newbler (version 2.9) as described [32]. Access to the unpublished draft and ongoing genomes analyzed in this paper can be obtained by contacting the corresponding author.

\section{Essential motifs and residues, alignments, and} phylogenetic analyses of PRAC sequences Predicted amino-acid sequences from PRAC genes identified in the trypanosome genomes were evaluated regarding motifs essential for racemase activity to identify putative PRAC homologous enzymes, thus ensuring the selection of genes encoding racemases, and excluding closely related PRAC-like genes such as those coding for epimerases. Previous studies on T. cruzi PRAC enzymes and bacterial PRACs demonstrated that catalytic cysteines (Cys130 and Cys300), active site (SPCGT) and essential motifs (MCGH and MIII) are not sufficiently stringent to discriminate between PRAC-like enzymes such as hydroxyproline-2 epimerase (HyPRE) and racemase. Therefore, the residues R1, R2, and R3, which are involved in substrate specificity, were used to distinguish between PRAC from HyPRE enzymes in silico $[7,8,10,16]$. These features were examined to select the genes encoding putative homologous PRAC enzymes in trypanosome genomes.
Amino-acid and nucleotide sequences of whole TcPRAC-homologous genes ( 1062 bp) from the various trypanosome species were obtained from genome data banks, aligned using Clustal X v2.0 and manually adjusted. In addition, partial ( 1015 bp) PRAC nucleotide sequences obtained by PCR-sequencing were used for polymorphism analysis within T. cruzi by comparing sequences from TcITcVI and Tcbat isolates. An alignment was created with partial PRAC amino acid sequences from $T$. rangeli isolates of lineages A-E and T. conorhini as outgroup.

Maximum-likelihood (ML) and maximum-parsimony (MP) analyses were performed respectively with RAxML v7.2.8 and PAUP"v4b10 based on nucleotide and amino acid alignments. The MP tree search and bootstrap analysis were done using 500 replicates of random addition sequence swapped using TBR. The ML analysis employed GTRGAMMAI with 500 maximum parsimony starting trees. Model parameters of ML analysis were estimated over the tree search and bootstrap support was estimated with 1000 replicates in RAxML using maximum parsimony as starting trees and optimized in the best tree as previously described [38-43].

To compare the highly conserved TcPRAC genes from all DTUs, a network genealogy was inferred using nucleotide sequences and the neighbor-net method with Kimura's 2-parameter model implemented in SplitsTree4 V4.10 as described previously $[28,44]$. Internode support was estimated by performing 100 bootstrap replicates using the same parameters optimized for network inferences.

\section{PCR amplification and sequencing of PRAC gene} sequences from $T$. cruzi and $T$. rangeli isolates

PCR amplification of partial TcPRAC sequences ( 1015 bp comprising all essential motifs and residues of TcPRAC enzyme) from large number of isolates from $T$. cruzi and $T$. rangeli (Additional file 1) was conducted as previously described [8] using the primers PRAC1 (5'-CTTCCCATGG GGCAGGAAAAGCTTCTG-3') and PRAC2 (5'-CTGA GCTCGACCAGATCTATCTGC-3'). The PCR-amplified products were cloned, and 3-5 clones from each isolate 
were sequenced, whereas $\sim 10$ clones were sequenced from each of the hybrid isolates. The PRAC sequences representing the genetic diversity within $T$. cruzi and $T$. rangeli were deposited in GenBank and the access numbers are listed in Additional file 2.

Phylogenetic analysis based on gGAPDH gene sequences Phylogenetic tree of Euglenozoa species based on gGAPDH gene sequences was inferred by ML and MP as described above for PRAC genes. The alignment created for this analysis was done using for guidance a comprehensive alignment of kinetoplastid gGAPDH genes [3] and included sequences from 32 trypanosome species, non-trypanosome trypanosomatids of seven genera, and five bodonids and euglenids as outgroups. Bootstrap support was estimated with 100 pseudoreplicates in RAxML using GTRGAMMA. The Genbank access numbers of all gGAPDH genes included in the phylogenetic trees are listed in Additional file 1.

\section{Trypanosomes lacking PRAC genes as determined by genome search and/or PCR amplification}

The absence of PRAC genes in the genomes of T. brucei ssp. and T. evansi was confirmed by negative results in PCR tests of additional isolates of each species. Besides the lack of PRAC homologs in the genome of T. congolense IL3000 (subgroup Savannah), results were also negative for all other members of Nannomonas tested: T. congolense Cam22 (Forest), WG5 (Kilifi) and TREU1475 (Savannah), T. simiae and T. godfreyi [28]. DNA samples from these trypanosomes were kindly provided by Wendy Gibson, Bristol University, UK.

\section{Horizontal-gene-transfer analysis}

The horizontal-gene-transfer (HGT) analysis includes a comprehensive dataset of 2,530 PRAC-like protein sequences from prokaryotes and eukaryotes in the non redundant (NR) database. A BLASTp search was performed with a maximum-expected-value threshold of 1e-20, using the TcPRACA and TcPRACB sequences as queries. The retrieved sequences were checked for PRAC-like domains using the Batch search tool in the Conserved Domain Database (http://www.ncbi.nlm.nih.gov/ Structure/bwrpsb/bwrpsb.cgi). Multiple-sequence alignment was performed using MUSCLE v3.8, and edited using Gblocks v0.91b [45] to eliminate poorly aligned positions. The final phylogenetic tree was obtained by $\mathrm{ML}$ analysis with 2,530 sequences under the WAG substitution model with gamma-distributed heterogeneity rate categories, and estimated empirical residue frequencies (model PROTGAMMAWAG) as implemented in RAxMLv7.2.8. One hundred different best tree searches were performed, and the tree with best likelihood found was kept. RAxML rapid bootstrap was performed with
100 pseudoreplicates. The tree was also visualized using Dendroscope v3.2.4 [46] with further cosmetic adjustments done using the Inkscape vector image editor (http://inkscape.org). To better resolve and visualize the putative HGT donor lineages, a ML analysis was performed using a subset of 303 PRAC-like sequences from NR database and 39 TryPRAC aligned with the nearest neighbor taxons identified in the analysis using 2,530 sequences. The tree search and bootstrap were conducted using the same parameters for both datasets.

\section{Genomic organization, GC content and codon pressure analyses of trypanosome PRAC genes}

The comparison of PRAC genomic organization in the analyzed genomes was performed with the bl2seq BLASTX algorithm using the flanking downstream and upstream regions $(\sim 10,000 \mathrm{bp})$ previously reported for T. cruzi and T. vivax [8,9] in all trypanosome genomes investigated in this study. Codon-selection analysis was performed using the HyPhy v2.2 package [47] with a threshold $p$-value $<0.05$. The $\mathrm{GC}$ content comparison between TryPRAC homologs and both flanking genes and whole genomes were conducted using the mfsizes v. 1.8.3 software (http://sourceforge.net/projects/mfsizes/).

\section{Results and discussion}

Analysis of kinetoplastid and euglenid genomes shows TCPRAC homologs exclusively in Trypanosoma

We searched for TcPRAC homologs in the genomes of trypanosomatids, bodonids and euglenids using TcPRACA and TcPRACB sequences as queries. Homologs were identified in T. cruzi, T. c. marinkellei, T. dionisii, T. erneyi, T. rangeli, $T$. conorhini and $T$. lewisi. In addition to these mammalian parasites, TcPRAC homologs were found in trypanosomes from snake (T. serpentis) [42], crocodile [43,48], lizard (TCC878), bird (TCC1825) and toad (TCC339) [41] (Table 1, Figure 1). All these species exhibit a single copy of a TryPRAC homolog per haploid genome, and no other PRAC-like gene was identified in the kinetoplastid genomes.

Previous studies reported the absence of PRAC homologs in the genomes of T. b. brucei and T. b. gambiense, while only a remnant of a PRAC gene was suggested for $T$. congolense [8-10]. Thus, it was not surprising that $T$. evansi, which is highly closely related to $T$. brucei ssp. and T. equiperdum, all forming the subgenus Trypanozoon, also lacked PRAC. In addition, attempts to detect even small fragments of PRAC genes by PCR amplification failed for all investigated species of the subgenus Nannomonas: T. congolense of subgroups Forest, Kilifi and Savannah, $T$. simiae and $T$. godfreyi. We were also unable to detect any PRAC fragment in the recently assembled genome of $T$. congolense (Savannah). In the phylogenetic trees, the species of Trypanozoon and Nannomonas formed 


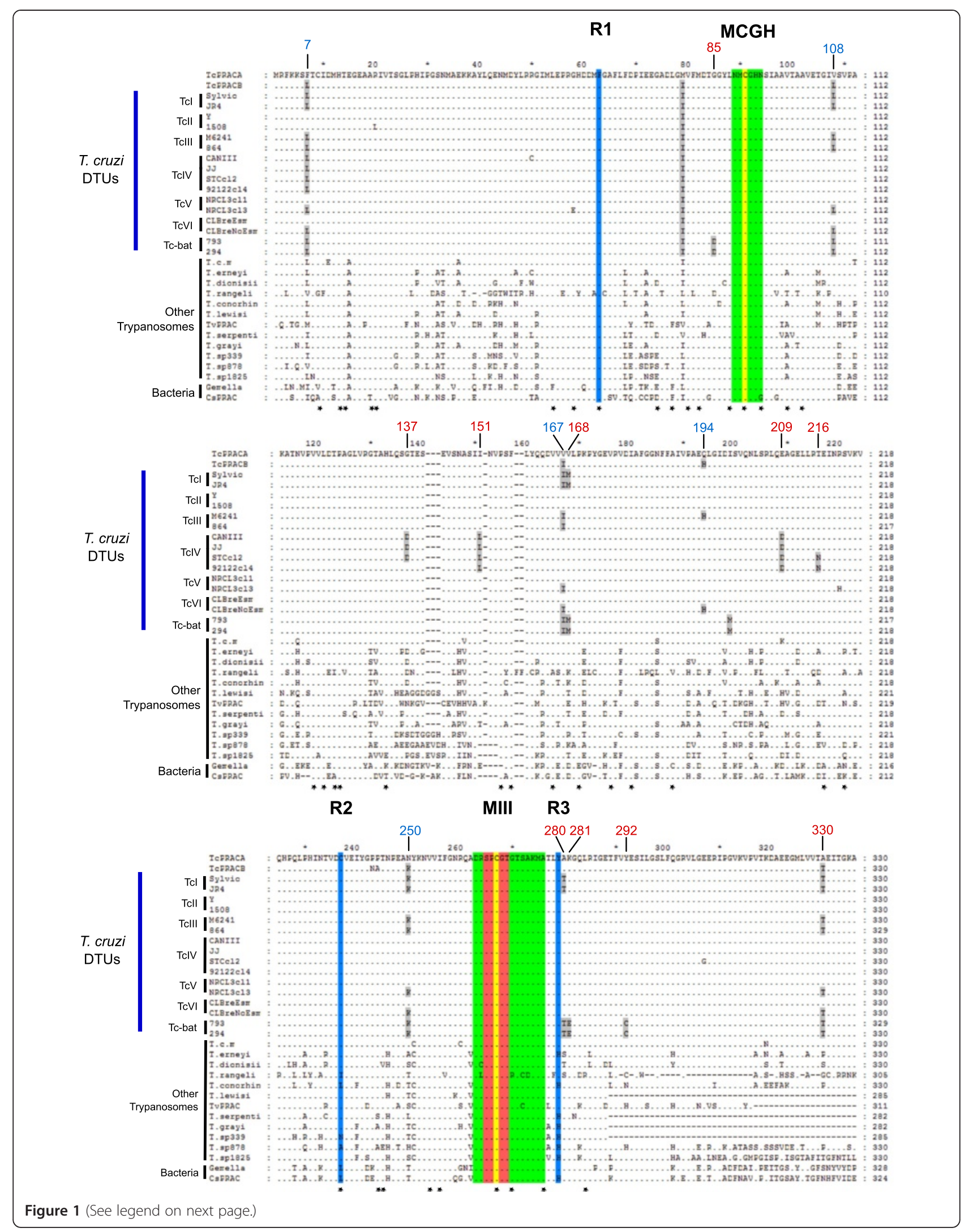


(See figure on previous page.)

Figure 1 Alignment of predicted amino acid sequences of proline racemase (PRAC) homologous genes from T. cruzi (DTUs TCl-TcVI and Tcbat), T. cruzi marinkellei (T. c. m), T. erneyi, T. dionisii, T. rangeli, T. conorhini, T. lewisi, TvPRAC - T. vivax, T. serpentis, T. grayi, T. sp. from toad (TCC339), T. sp. from lizard (TCC878), and T. sp. from bird (TCC1825) and PRAC from Gemella haemolysans and Clostridium difficile (CsPRAC). Essential motifs (MCGH and MIII) are in green, and the active site (SPCGT) in red. R1, R2 and R3 are residues involved in substrate specificity. Cys91 and Cys267 are the catalytic cysteines. Blue numbers indicate differences between TCPRACA and TCPRACB, and red numbers indicate substitutions found in newly identified TryPRAC homologs. Black stars indicate negatively selected amino acid residues.

a monophyletic assemblage within the main $T$. brucei clade in which T. vivax has a basal position [3].

Examination of non-trypanosome Trypanosomatidae species revealed that PRAC genes, and even other genes of the PRAC-like family, are absent not only from the $L$. major genome as previously reported [8-10,31], but also from other Leishmania species. Moreover, the genomes of monoxenous parasites of insects of the genera Crithidia, Leptomonas, Angomonas, Strigomonas and Herpetomonas and the plant parasites of the genus Phytomonas all lacked PRAC. Regarding other kinetoplastids, our searches did not reveal any putative PRAC-like genes in Bodo sp. and Parabodo caudatus. In addition, we did not identify PRAC-like genes in the genomes of the basal species within Euglenozoa: Euglena gracilis, Eutrepia viridis and Discoplastis spathirhyncha (Figure 2B).

In conclusion, PRAC homologs are widespread in trypanosomes but absent from the genomes of nontrypanosome trypanosomatids of all genera investigated, and in the bodonids and euglenids to date examined. PRAC homologs were identified in species of the main clades of the Trypanosoma phylogenetic tree, including the basal Aquatic clade [3,41], but in the T. brucei clade only $T$. vivax carries a homologous PRAC gene. Results strongly suggest that a prokaryotic PRAC was introduced at the root of Trypanosoma and fixed in the genome of a common trypanosome ancestor.

\section{Molecular characterisation of new putative PRAC-like enzymes of trypanosomes}

The catalytic mechanism of TcPRAC is essentially identical to that of the prokaryotic PRAC enzymes. The activity of PRAC enzymes depends mainly on two cysteine residues that transfer protons to the chiral carbon $\left(\mathrm{C}^{\alpha}\right)$ of L-proline/ D-proline enantiomers, resulting in the stereoinversion of its configuration $[7,10,16]$. PRAC-like genes include diverse racemase-like genes that exhibit strong sequence similarity to proline racemases. A few eukaryotic PRAC-like enzymes have all the residues critical for racemase activity. Most of these enzymes function as proline epimerases, which are common in prokaryotes or as proline dehydratases as reported in humans [16,17].

Alignment of TryPRAC homologs from 13 trypanosome species, using TcPRACs and prokaryotic PRAC sequences for guidance, revealed the two cysteine residues, the active site SPCGT, and the MCGH motif in all sequences. Only the MIII motif had relevant polymorphism. At the residues RI, R2, and R3, which are involved in substrate specificity, all TryPRAC homologs have a conserved R1 but a variable R2 and R3. All species of the subgenus Schizotrypanum (T. cruzi, T. c. marinkellei, $T$. dionisii and $T$. erneyi) share identical motifs, and highly conserved essential residues. Within this clade, only $T$. erneyi showed one non-synonymous substitution at R3. In contrast, more distantly related trypanosomes showed synonymous and non-synonymous substitutions at R2 (T. rangeli, T. conorhini, $T$. sp. from toad and $T$. sp. from lizard) and R3 (T. conorhini, $T$. serpentis, $T$. grayi and $T$. sp. from toad) (Figure 1). The implications of these substitutions in substrate specificity merit further investigation.

All putative TryPRAC sequences lacked a signal peptide, suggesting that the encoded enzymes are intracellular, and can be released through the flagellar pocket and/ or parasite death $[8,10,18]$. Despite highly conserved catalytic domains, we identified at least one novel PRAC homolog for each species of trypanosome. In silico analysis suggested that most trypanosomes can express PRAC proteins with racemase activities (Figure 1). Homologous PRAC of T. vivax differed in several residues when compared to those from T. cruzi. Although the PRAC genes from trypanosomes of non-mammalian hosts such as snakes ( $T$. serpentis), crocodiles ( $T$. grayi), toads, lizards and birds differed in several residues when compared to homologs of Schizotrypanum species, all sequences could be aligned with confidence with both TcPRAC and prokaryotic PRAC genes (Figure 1).

\section{Phylogenetic relationships of PRAC homologs from 13 trypanosome species agree with the currently recognised phylogeny of Trypanosoma}

T. cruzi is highly closely related to all other species of the subgenus Schizotrypanum (T. c. marinkellei, T. dionisii and T. erneyi), which are all called T. cruzi-like because they share morphology of blood and culture forms, although they differ in hosts, vectors and pathogenicity. Development as amastigotes and differentiation into trypomastigotes within mammalian cells in vitro is a unique feature of Schizotrypanum, whereas in vivo, only 
B. gGAPDH

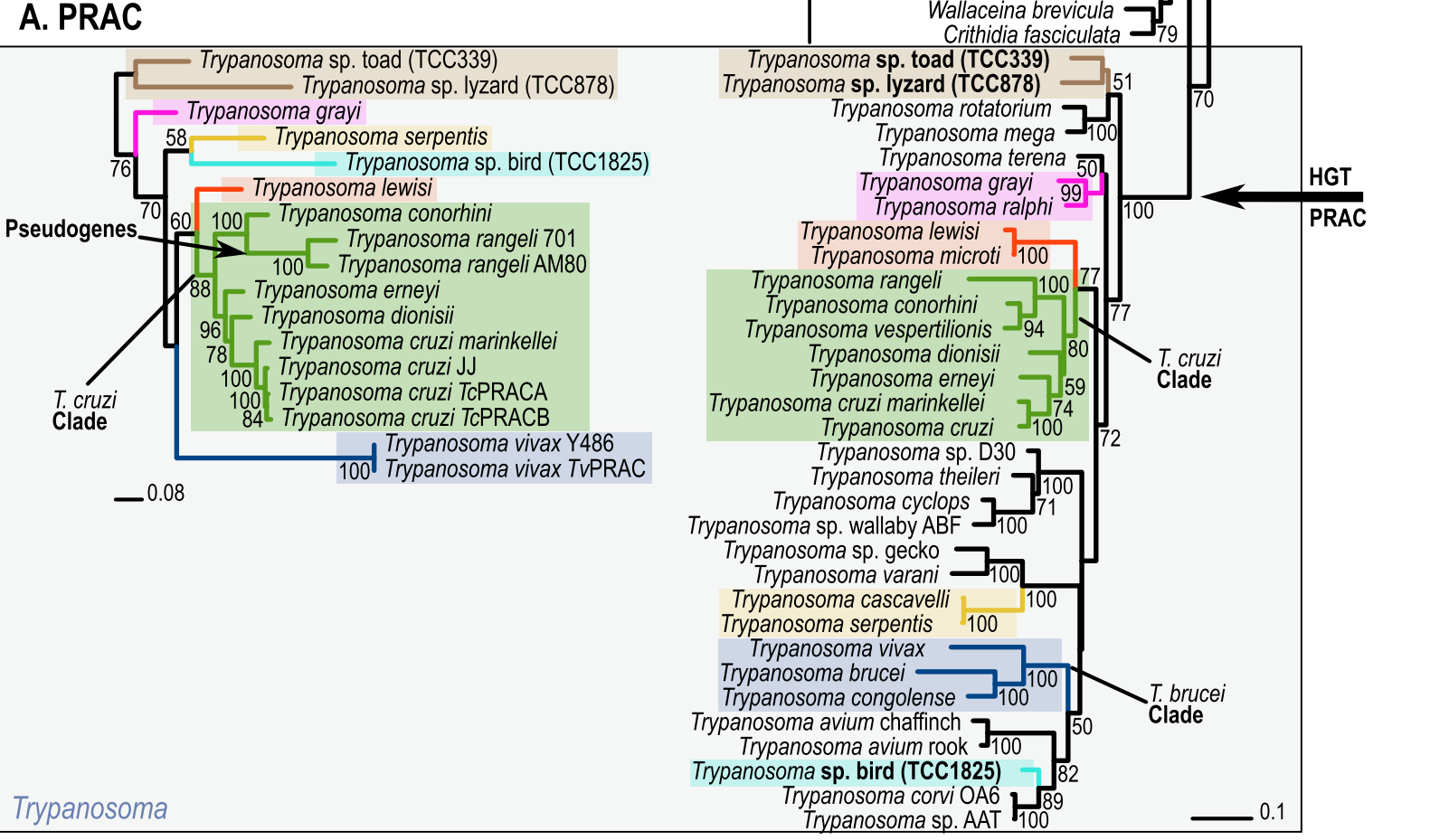

Figure 2 Congruent phylogenies of trypanosome species inferred using sequences from PRAC (A) and gGAPDH (B) genes. Maximum likelihood phylogenetic trees were inferred using entire TryPRAC nucleotide sequences from 13 trypanosome species $(-\mathrm{Ln}=8403.576084)$, and gGAPDH sequences from 32 trypanosome species, non-trypanosome trypanosomatids of seven genera, and bodonids and euglenids as outgroups $(-\operatorname{Ln}=13563.555163)$. Numbers on branches represent bootstrap support $(>50)$ estimated with 100 pseudoreplicates in RAxML using GTRGAMMA. The arrow on the PRAC genealogy (A) indicates pseudogenes found in T. rangeli. The arrows on the gGAPDH tree (B) indicate the places hypothesised for the horizontal transference of bacterial PRAC gene to a common ancestor of Trypanosoma, and the gene loss in a common ancestor of T. brucei ssp. and T. congolense. Genbank accession numbers of PRAC and gGAPDH gene sequences are respectively showed in Table 1 and Additional file 1.

T. cruzi infects mammals other than bats. Nevertheless, as in T. cruzi infection, nests of amastigotes in cardiac cells can be found in bats infected with T. cruzi-like species. $T$. cruzi is transmitted by triatomines, while cimicids are vectors of $T$. dionisii. These trypanosomes share development restricted to the vector guts [5,38-40,44].

For phylogenetic inferences within Schizotrypanum, we compared isolates of all species mentioned above. According to strongly supported branching patterns on both PRAC (Figure 2A) and gGAPDH (Figure 2B) phylogenetic trees, all species clustered tightly, forming a monophyletic assemblage of trypanosomes. The relationships among the Schizotrypanum species and the
DTUs of $T$. cruzi were inferred using entire PRAC amino acid sequences. Results corroborated the clustering of sequences according to species (Additional file 3). In agreement with previous analysis of several other genes [49] such as gGAPDH and cathepsin L-like genes, TryPRAC genealogy and nucleotide sequence divergences confirmed T. c. marinkellei as the closest relative of $T$. cruzi ( 7.5 of TryPRAC sequence divergence between the two species). This species was followed by $T$. erneyi from African bats ( 14\%) and T. dionisii (14.5\%) from Old World bats. Large genetic distances separated $T$. cruzi and T. rangeli ( $30 \%$ PRAC sequence divergence), and $T$. cruzi and T. vivax ( 38\%) PRAC 
sequences. Compared to divergence among TryPRAC sequences, the species of the subgenus Schizotrypanum were separated by much smaller gGAPDH sequence divergences $(\sim 8.0 \%$ between $T$. cruzi and $T$. dionisii), whereas $\sim 15 \%$ and $\sim 17.5 \%$ of gGAPDH sequence divergence separated T. cruzi from $T$. rangeli and T. vivax, respectively. Divergences of gGAPDH and the more conserved SSU rRNA genes, which are the traditional genes employed for phylogenetic inferences of the Trypanosomatidae, were previously reported for the trypanosome species included in PRAC phylogeny $[5,39,40]$.

In the bat-seeding hypothesis for the origin of the $T$. cruzi clade [39], a scenario has been proposed in which ancestral trypanosomes of bats evolved exclusively in Chiroptera, giving rise to the bat-restricted species [38-40], or evolved through multiple independent host jumps, giving rise to species infecting other mammals (such as rats, civets and monkeys) in the Old World, and to the generalists $T$. cruzi and $T$. rangeli, which are species infective to bats plus a broad range of other mammals, including human and non-human primates in the New World [3,5,38-40,50-53]. Also in agreement with previous studies, in the PRAC genealogy $T$. rangeli and T. conorhini formed the sister group of Schizotrypanum, together constituting the clade T. cruzi that also harbours other trypanosomes, mostly from bats $[39,40]$. T. lewisi, the basal species of the clade T. cruzi, is a nonpathogenic and cosmopolitan trypanosome of domestic rats transmitted by fleas, which can opportunistically infect immune-compromised human and non-human primates [54] (Figures 2A, 2B).

The toad and lizard trypanosomes included in PRAC genealogy represented the basal branches of the Trypanosoma gGAPDH phylogenetic trees showing PRAC genes highly divergent from TcPRAC (Figure 2A). Previous $[3,41-43,48]$ and herein inferred phylogenies based on gGAPDH genes (Figure 2B) demonstrated that anuran trypanosomes nested into the so-called "Aquatic clade", which also includes trypanosomes transmitted by aquatic leeches of fishes, turtles and platypus, besides a lizard trypanosome of unknown vector. The aquatic clade was strongly supported as the most basal of Trypanosoma [3]. Also concordant with previous phylogenies, PRAC genes of trypanosomes from snakes, birds and crocodiles, which are transmitted by insects, all clustered into the "terrestrial" clade that also includes PRAC sequences from all trypanosomes of mammals (Figure 2A) in agreement with previous SSU rRNA and gGAPDH phylogenies [3,41-43,48].

\section{Repertoires and phylogenetic relationships among T. cruzi PRAC homologs of all DTUs and Tcbat}

Comparison of whole TryPRAC amino acid sequences revealed relevant polymorphisms $(\sim 3.0 \%$ sequence divergence) within T. cruzi. Aiming an intra-specific analysis of TcPRACs, we compared entire amino acid sequences from T. cruzi Sylvio X10.6, JRcl4 and G (TcI), Esmeraldo cl3 (TcII), M6241cl6 (TcIII), CANIII (TcIV), CL Brener (TcVI), Tula (TcVI), and Tcbat. Unlike all other isolates, which carried a single PRAC gene, T. cruzi CL Brener exhibited TcPRACA and TcPRACB [10], found in this work in the Esmeraldolike and non-Esmeraldo-like haplotypes, respectively.

We evaluated all signatures defined for TcPRAC activity, and polymorphisms used to differentiate between TcPRACA and TcPRACB. A leucine at position seven (typical of TcPRACB) was found in TcI, TcIII, TcIV and Tcbat. The phenylalanine at this position that had been reported to be specific to TcPRACA was found in $\mathrm{Y}$ and TCC1508 (TcII), but not in Esmeraldo (TcII). TcPRACs from all DTUs have isoleucine at position 79 (like TcPRACB), while methionine at this position in TcPRACA was exclusive to CL Brener. Like TcPRACA, TcII and TcIV had valine at positions 108 and 167 and asparagine at position 250. At these positions, TcI, TcIII and Tcbat had leucine, isoleucine and lysine, respectively, like TcPRACB. Like the hybrid $\mathrm{CL}$ Brener (TcVI), both TcPRACA and TcPRACB were identified in the hybrid NRCL3 (TcV). New polymorphic residues evidenced novel TcPRAC homologs defining TcI-TcIV-specific profiles while $\mathrm{TcV}$ and $\mathrm{TcVI}$ can be identified by the presence of both TcPRACA and TcPRACB. Polymorphic amino acids defining each T. cruzi DTU are showed in Additional file 3.

Due to the high sequence conservation throughout the TcPRAC genes from T. cruzi of some DTUs, phylogenetic analyses based on amino acid sequences were unable to clearly resolve the closely related DTUs (Additional file 3 shows the network of TcPRAC amino acid sequences). To assess the relationships within T. cruzi using the conserved TcPRAC genes (63 polymorphic sites), we constructed a network using partial nucleotide sequences obtained by PCR-sequencing from 68 isolates previously genotyped $[21,44,49]$. The network clearly evidenced subclades corresponding to each TcI, Tcbat, TcII, TcIII, and TcIV DTUs. Sequences from $\mathrm{TcV}$ and TcVI clustered with TcII or TcIII, in agreement with their hybrid origin, forming a reticulate pattern in the network. The network confirmed TcI closest to Tcbat and TcII more related to TcIV and, in addition, corroborated the heterogeneity intra-DTUs TcI, TcIII, and TcIV (Figure 3).

Most previous phylogenetic analyses within T. cruzi lacked isolates of all DTUs (especially TcIV and Tcbat) and/or T. cruzi-like outgroups and, then, were insufficient to resolve intra-T. cruzi phylogenetic relationships. Here, TcPRAC genealogy using T. cruzi-like species agreed with the relationships among the DTUs (including Tcbat) previously inferred using cruzipain, SSU rRNA, and cytochrome b sequences [44,49]. Diverse 


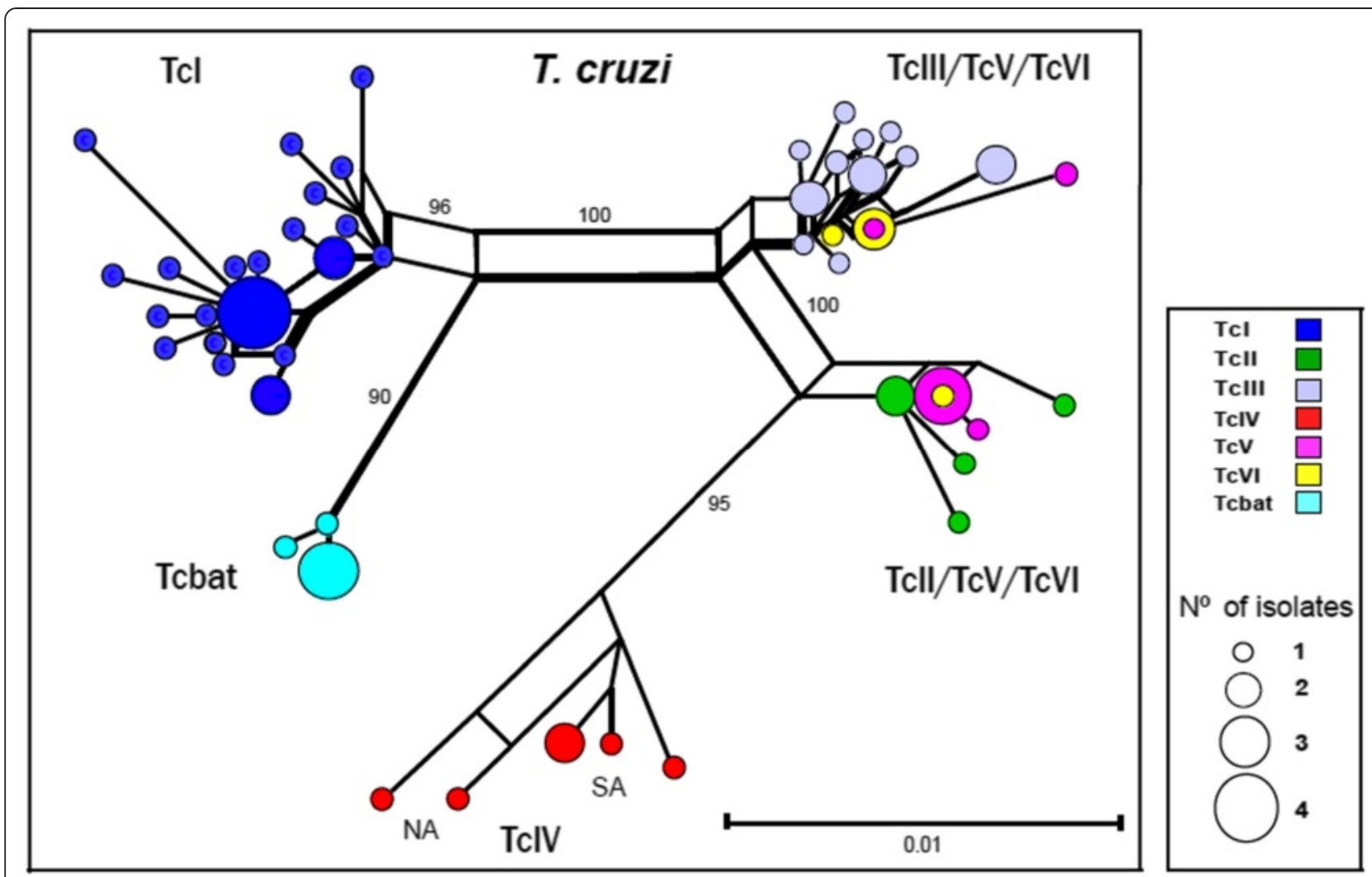

Figure 3 Network genealogy of partial PRAC nucleotide sequences from T. cruzi isolates showing the clustering of sequences according to DTUs, and sequences from the hybrids TCV and TcV positioned within both Tcll and Tclll clades. The network branching pattern indicated high homogeneity of PRAC genes from isolates of Tcbat, Tcll and Tclll contrasting with the large heterogeneity in Tcl and TcIV isolates. Each colour represents one DTU, and the size of the circles indicates the number of isolates in the network. Genbank accession numbers of TCPRAC genes are listed in Additional file 2, and the DTU-specific profiles of polymorphic sites is showed in Additional file 3.

genes have been employed as markers for T. cruzi genotyping [21]. We demonstrated that polymorphisms of TcPRAC sequences allow the genotyping of all DTUs including hybrid genotypes, and are also valuable to infer inter-DTU relationships. To our knowledge, TcPRAC is the first horizontally transferred gene (non-mitochondrial) characterized with these purposes.

Taking into account that TcPRAC-A and TcPRAC-B participate in $T$. cruzi development in vertebrates and vectors and have been incriminated as host defence factors [9-12,19,22,55], it is tempting to speculate whether DTU-specific TcPRAC enzymes can contribute to differential degrees of metacyclogenesis, parasitemias and virulence. T. cruzi of different DTUs interacts differently with the host, induces distinct immune responses and infections ranging from highly lethal to virtually asymptomatic, contributing to variable clinical forms of Chagas disease. Strains of TcI, the most widespread DTU in Latin American sylvatic cycles, exhibit high levels of metacyclogenesis. TcI is responsible for human outbreaks of oral infection and severe cardiomyopathies in Central America and Northern
South America. Isolates of TcI are highly diverse genetically and in terms of virulence to mice. Although some isolates induced very low parasitemia and no mortality, it was suggested that Col strain ( TcI) can evade the host immune response remaining unnoticed by mononuclear cells allowing rapid multiplication during acute infection [21,50-53,56-60]. Future studies are required to evaluate the roles played by TcI-specific TcPRAC in low and highly virulent strains. TcII (PRAC-A), TcV and TcVI (PRAC-A and PRAC-B) are virulent to mice, induce high parasitemias and mortality, and have been associated with both cardiac and digestive forms in humans in Southern Cone countries [21,61]. TcIII strains (PRAC-B), found in Brazil and neighbouring countries, can induce important parasitemia and pathology in mice $[57,62]$. TcIV (unique TcPRAC) is sylvatic, orally infects humans in Brazil and Venezuela, and induces low or moderate parasitemia and mortality in mice $[21,53,60]$. Tcbat, found in South and Central American bats and, apparently, able to infect humans [63], is not virulent to mice inducing extremely low parasitemias and no mortality, and is unable to develop in the commonest triatomine vectors of TcI-TcVI [50]. 
Trypanosoma rangeli pseudogenes diverged to be lineage-specific and more closely related to $T$. conorhini than to $T$. cruzi homologous PRAC

$T$. rangeli is a non-pathogenic parasite of humans and domestic and wild animals in Central and South America. This species is thought to be restricted to the bloodstream and survives host defences for months or years by unknown mechanisms. T. cruzi and T. rangeli are the only agents of human trypanosomosis in the Americas, sharing mammalian hosts and vectors in overlapping areas. $T$. rangeli overcomes the defences of the vector (Rhodnius spp.), multiplying in the gut and invading the haemolymph, where the parasites multiply outside and inside of haemocytes before reaching the salivary glands where metacyclogenesis takes place. This species differs from $T$. cruzi, which develops exclusively in the triatomine gut, and from $T$. brucei, which reaches the salivary glands of the vector (tsetse flies) from the proboscid $[4,64,65]$.

Closer phylogenetic relationships of $T$. rangeli to $T$. cruzi than to T. brucei were strongly supported by comprehensive phylogenetic analysis based on diverse genes [3,5,38-40]. In addition, phylogenies based on PRAC (Figure 2A), gGAPDH (Figure 2B) and SSU rRNA genes have supporting $T$. rangeli more closely related to $T$. conorhini than to T. cruzi $[3,38,40]$. T. conorhini is a tropicopolitan species common in rats and transmitted by the also tropicopolitan Triatoma rubrofasciata [66,67]. This species shares features with both T. cruzi (development restricted to the gut of its triatomine vector) and $T$. rangeli (lack of both intracellular stages and pathogenicity to vertebrates). We are currently comparing the genomes of T. conorhini, T. rangeli and T. cruzi to better understand their relationships.

In contrast to predicted PRAC proteins in most trypanosomes including $T$. conorhini, which are compatible with the expression of racemases, all $T$. rangeli PRAC sequences were found disrupted by internal stop codons resulting in pseudogenes. This finding was confirmed in the PRAC found in the genome of T. rangeli AM80 (human isolate of basal lineage TrB from the Amazon region) and sequences from several isolates of all lineages determined by PCR-sequencing. Additional file 4 shows the alignment of $T$. rangeli PRAC pseudogenes.

We compared T. rangeli PRAC pseudogenes from 17 isolates of lineages TrA-TrE, all previously genotyped using other markers [49,50]. Previous phylogeographical studies suggest evolution within $T$. rangeli shaped by the coexistence of parasites with sympatric species of Rhodnius $[4,51,52,64,65]$. In this and previous studies based on SL, SSU rRNA, ITS rDNA, gGAPDH, and CATL sequences, $\operatorname{TrB}$ was always placed as the basal lineage of T. rangeli, whereas the relationships among the closely related TrA, C, D and E were far from resolved (Figure 4). In addition, an increasing genetic diversity within $T$. rangeli

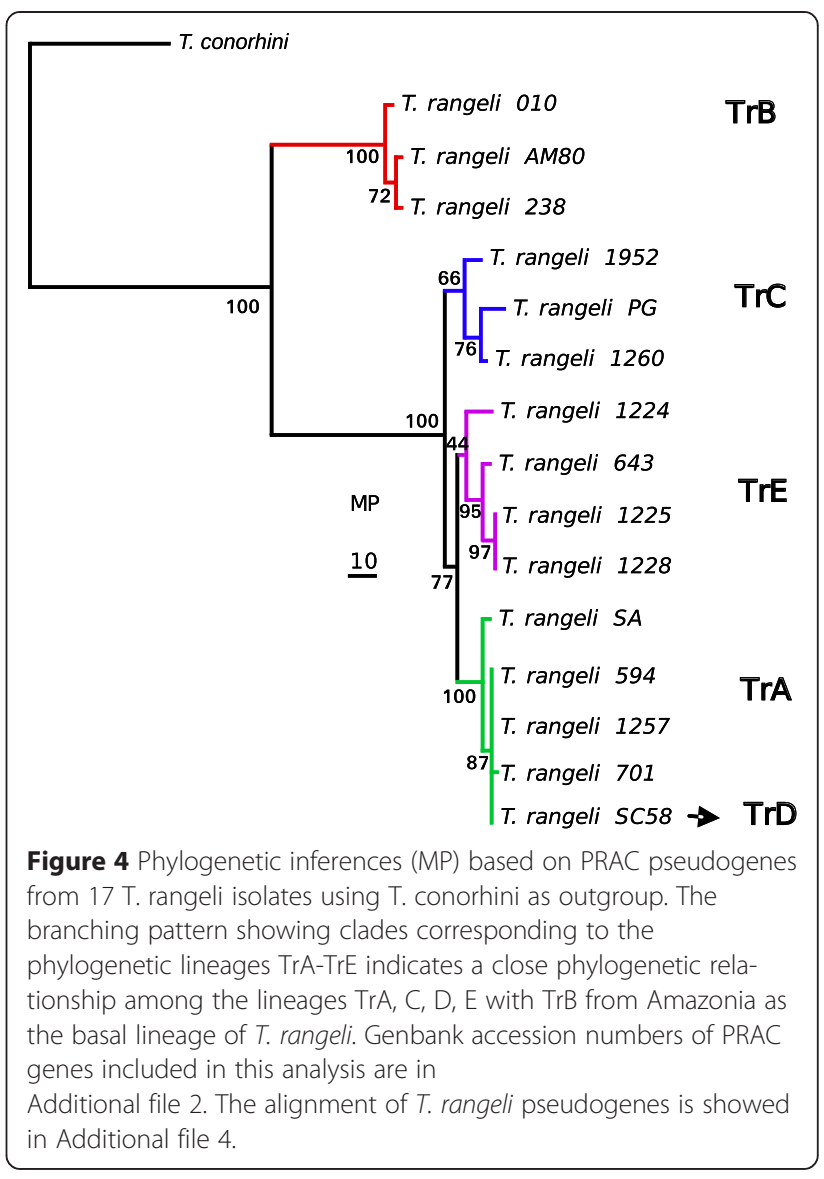

has recently been revealed $[51,52,63,64]$. Phylogenetic studies of all lineages using multilocus approaches are essential to better resolve the complex relationships among the lineages and to hypothesise about the evolutionary history of $T$. rangeli.

\section{Phylogeny and pattern of presence/absence of PRAC homologs support a single HGT from a bacterium to an ancestor of Trypanosoma}

The identification of bacterial PRAC homologs in $T$. cruzi and T. vivax and their absence from T. brucei and T. congolense [7-11] suggested a complex evolution of PRAC genes in trypanosomes. Here, a broad taxon sampling comprising 15 trypanosome species, trypanosomatids of 9 additional genera and five free-living ancestors of bodonids and euglenids provides relevant insights into this process at long and short timescales. The relative timing of the HGT event was investigated by searching the presence/absence of PRAC-like genes in the increased and broad taxon sampling. No PRAC-like genes were found in trypanosomes besides those encoding putative proline racemase enzymes, such as highly similar genes coding for epimerase and dehydratase found in prokaryotes and other eukaryotes such as fungi and metazoans. Although PRAC-like genes detected in fungi 
and trypanosomes were all likely of prokaryotic origin, previous phylogenetic analyses revealed a polyphyletic pattern, indicating that they originated from different bacterial sources through independent HGT events $[17,18]$. Here, no PRAC-like genes were detected in the genomes of euglenids, bodonids or non-trypanosome trypanosomatids.

After identifying in our taxon-rich phylogenetic analysis of 2,530 eukaryotic (including trypanosome) and prokaryotic PRAC-like genes (Figure 5A) the general vicinity of TryPRAC sequences (the names and grouping of all organisms can be found in the rectangular phylogram in Additional file 5), our targeted analysis of 342 genes showed all TryPRAC homologs in a strongly supported clade exclusive of trypanosome sequences, evidencing their common ancestry (Figure 5B). The complete list of putative donor lineages currently available on NCBI NR protein database included in the phylogenetic analysis is presented in Additional file 6.

Considering the presence/absence of PRAC genes and the congruence of all species in phylogenies based on PRAC and gGAPDH genes (Figure 2), we hypothesised gains and losses of PRAC genes during trypanosome evolution. In the most parsimonious evolutionary scenario, one prokaryotic PRAC gene was transferred through a single HGT to the root of the genus Trypanosoma in a common ancestor of this genus, which gave origin to all extant trypanosome species with respective TryPRAC homologs. The absence in T. brucei and T. congolense suggested the loss of PRAC gene by a common ancestor of the subclade comprising these species (Figure 2B). In an unlikely, less parsimonious scenario,
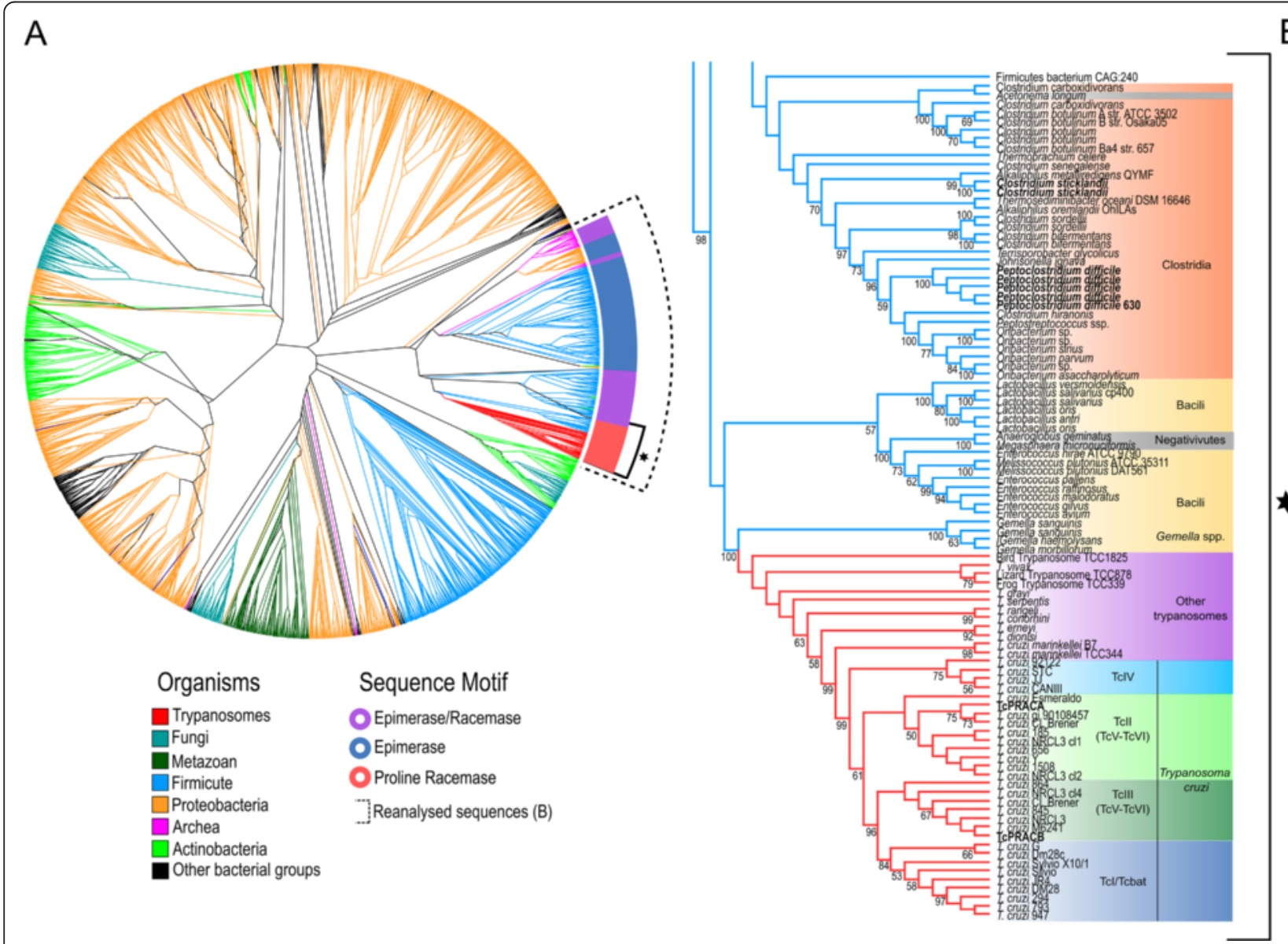

Figure 5 Phylogenetic analyses of PRAC-like protein sequences of prokaryotes and eukaryotes, from the NR NCBI database, and Try PRAC sequences. (A), ML analysis including 2,530 PRAC-like sequences with the best substitution model WAG + G. Bootstrap supports (100 pseudoreplicates) are displayed at nodes (only support of 50 or greater shown) in the phylogenetic tree, corresponding rectangular phylogram is showed in Additional file 5. The dotted line in (A) indicates the branch submitted to the reanalysis shown in Figure 5B. (B) ML inference restricted to TryPRAC (39 trypanosome species/isolates) plus 303 bacterial genes nearest relatives to TryPRAC homologs showing, closest to TryPRAC sequences, Firmicutes PRAC genes from species of Gemella, other bacteria of Bacilli class, and species of Clostridia class. The PRAC-like gene sequences employed in the phylogenetic analysis showed in Figures 5A and 5B are listed in Additional files 6 and 7, respectively. 
PRAC homologs were present in all trypanosomatids or even in more basal euglenozoans, and were lost by most lineages being retained exclusively in the genus Trypanosoma.

Together, phylogenetic analysis and the pattern of absence/presence of PRAC-like genes strongly support our HGT hypothesis. However, it is important to consider other alternative scenarios, such as insufficient taxon sampling (donor lineages not represented in the analysis) and convergent evolution, which is an important cause of misidentified orthologs $[68,69]$. However, convergent evolution is in general restricted to functional domains and not across the protein length and, then, was not suggested by the alignment of TryPRAC with PRAC homologs from Gemella haemolysans and Clostridum difficile (Figure 1).

\section{Taxonomy of TryPRAC donor prokaryotic lineages}

In the most robust approach to assess taxonomic affiliation of putative HGT donors, all the most likely candidates selected by BLAST searches (useful for a primary screen of potential donors) should be submitted to deep phylogenetic analyses [17,18,31-35,68-71]. Here, the results obtained using this approach corroborate previous phylogenetic studies of prokaryotic and eukaryotic PRAC-like genes, suggesting prokaryotic donors for TcPRAC and TvPRAC [8-10]. In addition, we provided new insights into the origin and evolutionary history of TryPRAC homologs. The finding of all TryPRAC homologs clustering tightly together in a monophyletic assemblage, distant from any PRAC-like genes of other eukaryotes and within a large clade of prokaryotic PRAC-like genes, corroborated a single bacterium as donor lineage (Figures 5A and 5B) [Additional file 5].

To better resolve the phylogenetic relationships and visualise the most likely TryPRAC donor lineages, 303 prokaryotic sequences adjacent to TryPRACs were employed for further ML analysis (Figure 5B). The full list of taxa included in this analysis is shown in Additional file 7. The results suggest that the donor was a bacterium related to species of the Bacilli class of the orders Lactobacillales (bacteria that live in soil, water, plants and animals) and Negativicutes (anaerobes that live in rivers, lakes and animal guts) [72], including species of the genera Gemella, Enterococcus, Lactobacillus and Melissococus (Figure 5B). After these species, PRAC homologs from species of Clostridium, Peptoclostridium and Oribacterium of the Clostridia class were the most closely related to TryPRAC genes. In previous studies $[8,10]$, the closest relatives of TcPRAC were Clostridium difficile (reclassified as Peptoclostridium) and Clostridium sticklandii, both Firmicutes of the Clostridia class [72]. In this analysis, PRAC homologs from Gemella haemolysans, G. morbillorum and G. sanguinis, all exhibiting typical residues of PRAC racemase enzymes, were the nearest relatives to TryPRACs ( 57\% of identity) (Figure 5B). The species of Gemella are oral and gastrointestinal commensals of animals including humans that, as opportunistic pathogens, cause severe pulmonary, cardiac and cerebral infections [73]. Interestingly, G. haemolysans and G. morbillorum are highly prevalent among the bacterial fauna of haematophagous dipterans of Culicidae [74], which can transmit trypanosomes among anurans [41].

\section{Synteny analysis revealed highly conserved gene order around TryPRAC homologs}

Our findings demonstrated that TryPRAC homologs are ubiquitous in the genus Trypanosoma (Figure 2A). Previous studies revealed high conservation of gene segments containing the PRAC locus from T. cruzi and T. vivax $[8,10]$. To verify the genome organisation of PRAC genes in the different trypanosome species, we performed BLAST searches in genome databases for orthologous genes in PRAC loci. The results showed a syntenic block shared by all species (Figure 6). The adjacent regions of PRAC genes exhibited high synteny in T. cruzi, T. $c$. marinkellei, T. erneyi, T. dionisii, T. vivax and T. grayi, with at least 8 genes arranged in the same order: K39 kinesin, WD domain-containing protein, cold shock domain-containing protein, PRAC, hypothetical protein, zinc-finger protein, poly (A) polymerase, carbohydrate kinase and phosphatidyl serine. Syntenic organisation strongly supports orthology, with a single insertion of a prokaryotic PRAC gene between the cold-shock and

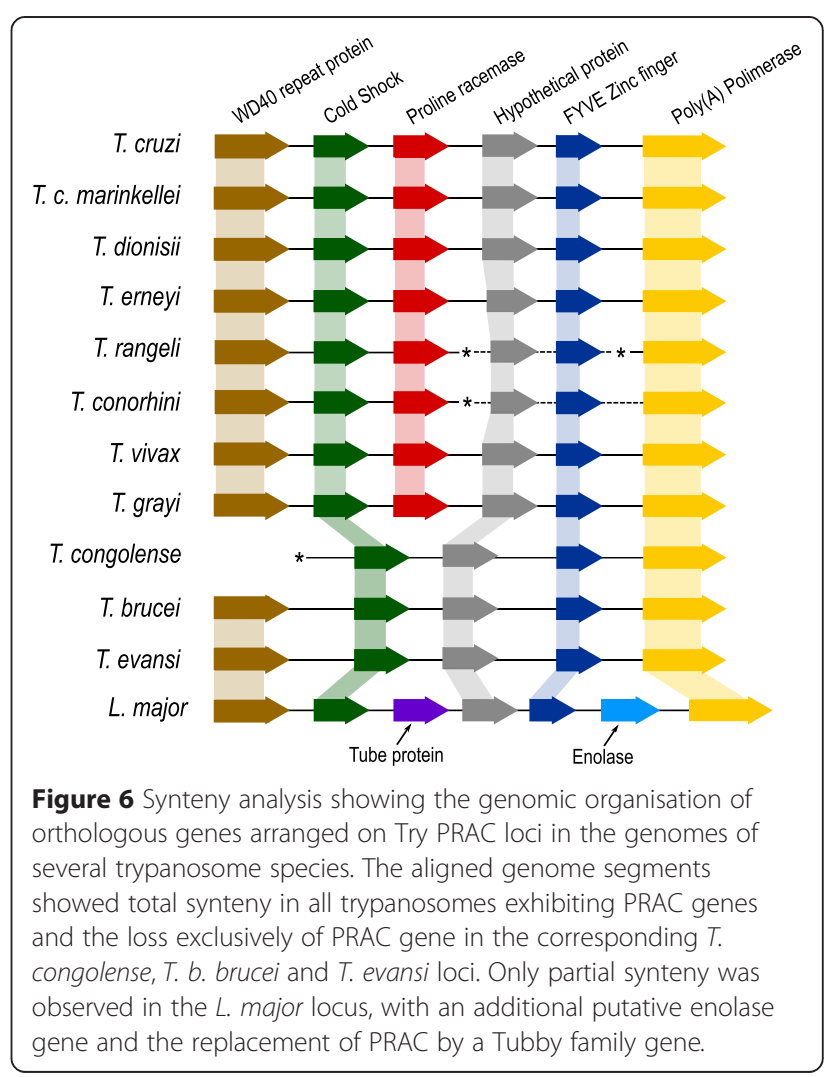


hypothetical protein genes. Although T. congolense and T. $b$. brucei lack PRAC genes, the synteny of this genome segment was retained in these species as well as in this locus of the T. evansi genome. These species nested into a single clade with $T$. vivax as the basal species. The synteny in PRAC loci corroborated the loss of PRAC by a common ancestor of these species after the divergence of $T$. vivax (Figure 2B). Figure 6 shows the conserved gene order of five orthologs flanking the gene PRAC of trypanosomes.

Synteny analysis requires larger contigs still not available in some ongoing genome drafts. Consequently, synteny was confirmed partially for $T$. rangeli and $T$. conorhini (Figure 6). Only the two upstream genes were found in $T$. serpentis, and no genes flanking PRAC genes could be confidently located in the genomes of the non-mammalian trypanosomes. The PRAC locus showed only partially syntenic orthologs in the available genomes of non-trypanosomes trypanosomatids. In Leishmania major and Crithidia spp., which are phylogenetically related organisms [1,2], the position occupied by the PRAC gene was taken by a Tubby superfamily gene. In addition, these species also exhibited a putative enolase gene absent in this segment of trypanosome genomes (Figure 6). The insect trypanosomatids Angomonas desouzai and Strigomonas culicis lack both PRAC and Tubby family genes, while conserving the other flanking genes (data not shown). Therefore, corroborating the high plasticity of trypanosomatid genomes, the gain and loss of the PRAC gene in trypanosome genomes was not the only event that occurred in this genome segment during the evolution of the trypanosomatids.

\section{PRAC homologs from trypanosomes are largely under the influence of purifying selection}

One common characteristic of HGT is that the horizontally acquired bacterial gene and host vertically inherited genes can differ in GC content and codon usage, depending on the relative timing of the HGT event [68-71]. Despite considerable GC content divergences among the genomes of different trypanosome species, the averages of the GC contents of whole genomes were comparable to those from TryPRAC and flanking genes, such as in the genomes of T. cruzi G ( 49, 48 and 48\%, respectively, for PRAC, PRAC loci and whole genome), T. dionisii ( 52, 55, 47\%), T. vivax ( 49, 49, 46\%) and $T$. grayi $(\sim 63,55,54 \%)$. Results indicating that the PRAC gene acquired by HGT has been strongly adapted to the codon usage of the host genes are consistent with ancient acquisition of the bacterial PRAC gene by one ancestor of Trypanosoma.

Protein sequences can continuously evolve under the effect of evolutionary pressure that arises as a consequence of the host-parasite interactions including host immune defences. To examine positive or negative selection pressures on the evolution of TryPRAC, we calculated the $\mathrm{dN} / \mathrm{dS}$ ratio for the putative TryPRAC homologs. Finding $\mathrm{dN} / \mathrm{dS}$ ratios below one, indicative of negative or purifying selection, suggested that positive selection is not the driving evolutionary force shaping the TryPRAC repertoire. In addition, codon selection was specifically investigated on TryPRAC motifs and residues essential for enzyme activity. The results indicated 40 negatively selected codons, 7 of which have known relevance to PRAC activity in T. cruzi and T. vivax (Figure 1), and no positively selected codon. PRAC homologs, despite evolving to be species and genotype specific, are largely under the influence of purifying selection. The fixation and evolution of PRAC genes under strong constraint in several trypanosomes suggests that PRAC activity should be advantageous to these parasites.

The genetic polymorphism analysis should be one of the first steps in the selection of promising vaccine and drug candidates [20]. A target sharing high conservation of all essential motifs in isolates representing the T. cruzi genetic repertoire, as evidenced previously for cruzipain [44] and herein demonstrated for TcPRAC enzymes are good candidates for a multivalent drug against Chagas disease.

\section{Conclusions}

T. cruzi and T. vivax PRAC enzymes are potent host B-cell mitogens that delay specific immune defences through the generation of non-specific B-cell proliferation, allowing parasite evasion and disease progression. These enzymes have also been linked to metabolism and parasite multiplication and differentiation. We identified TryPRAC homologs in the genomes of 12 trypanosome species, including newly sequenced genomes from trypanosomes of mammals, birds, snakes, lizards, crocodiles and toads. TryPRAC homologs were identified in most trypanosomes, including pathogenic and non-pathogenic species with different life cycles in vertebrates and vectors. $T$. brucei ssp., T. evansi, T. congolense, $T$. simiae and $T$. godfreyi, which are all pathogenic for mammals, were so far the only trypanosomes that lost PRAC genes.

The TryPRAC genealogy is congruent with the recognised relationships within Trypanosoma, with genes evolving to become species-specific and genotypespecific. A taxon-rich phylogenetic analysis strongly supports a bacterial origin for these genes. The presence of TcPRAC homologs in trypanosomes of the Aquatic clade (basal in the phylogeny of Trypanosoma) and the absence of any PRAC-like gene in other trypanosomatid genera, bodonids and euglenids, together with the high synteny of PRAC gene neighbourhood allowed us to hypothesise that a common ancestor of Trypanosoma gained the bacterial gene through a single HGT. Our analysis supports a Firmicutes bacterium as the donor lineage, and suggested that the closest 
relatives of TryPRACs are not in the genus Clostridium of Clostridia class as previously hypothesized, but more likely in the genus Gemella of the Bacilli class. However, this conclusion can change as more and more genome sequences become available in this important bacterial group. The results revealed unique PRAC homologs for each species as well as for each $T$. cruzi DTU and T. rangeli lineages. According to in silico analysis, all newly identified putative TryPRAC genes likely express functional racemases except $T$. rangeli, which has only pseudogenes. Together, our results suggest evolutionarily driven rearrangements on TryPRAC loci resulting in the fixation of intact PRAC genes in most trypanosomes, complete loss by the subclade T. bruceiT. congolense, and PRAC genes, apparently, in process of being lost in $T$. rangeli.

An understanding of the repertoire and evolutionary history of genes encoding TryPRAC homologs in a range of trypanosome species and genotypes can help understand the potential role of PRAC enzymes in hosttrypanosome interactions. Further analyses are required to evaluate the expression and any involvement of novel putative TryPRAC enzymes in the life cycles, infection strategies, pathogenicity, virulence, and host immune evasion of the various trypanosome species.

\section{Additional files}

Additional file 1: Table containing the isolates of $T$. cruzi and $T$. rangeli and respective host species, geographic origin and lineages, and Genbank accession numbers of their respective PRAC gene sequences.

Additional file 2: Genbank accession numbers of gGAPDH gene sequences included in the Figure $2 \mathrm{~B}$.

Additional file 3: (A) Network genealogy of entire PRAC amino-acid sequences from $T$. cruzi isolates of different DTUs. Colors represent the DTUs, and the size of circles indicates the numbers of isolates. (B) Polymorphic amino acids detected on the alignment comprising 68 partial PRAC sequences from T. cruzi isolates of TCl-TCVI DTUs and Tcbat.

Additional file 4: Alignment of predicted amino acid sequences of PRAC pseudogenes from $T$. rangeli isolates of all phylogenetic lineages (TrA-TrE). Essential motifs (MCGH and MIII) are in green, active site in red (SPCGT), R1, R2 and R3 in blue and Cys91/267 in yellow. Red asterisks indicate stop codons.

Additional file 5: Maximum likelihood phylogeny of 2,530 PRAC-like protein sequences (Figure 5A) displayed as rectangular phylogram.

Additional file 6: Genbank acession numbers of 2,530 PRAC-like family genes from prokaryotes and eukaryotes retrieved from full NCBI NR database and included in the Figure 5A.

Additional file 7: Genbank accession numbers of 303 prokaryotic PRAC-like genes closest related to TryPRAC genes retrieved from full NCBI NR database and included in the Figure 5B.

Competing interests

The authors declare that they have no competing interests.

\section{Authors' contributions}

ZEC, AGCM and RCF performed the sequencing, genome and phylogenetic analyses and participated in the manuscript preparation. JMPA, MGS and GB were responsible for genome sequencing of draft genomes within the ATOL project. PM, JMPA, GB and EPC contributed with data interpretation, discussion and manuscript preparation. MMGT designed and coordinated the study and drafted the manuscript. All authors contributed with discussions and have read, revised and approved the final version of the manuscript.

\section{Acknowledgements}

We are grateful to The Wellcome Trust (TriTrypDB) and to the Kinetoplastid Genome Sequencing and Analysis Consortium NIH/NHGRI/NIAID (project ID 59941) of the Genome Institute at Washington University School of Medicine (USA) for making genomes of trypanosomatids freely available. Additional draft and ongoing genomes were obtained within ATOL (Assembling the tree of Life - Phylum Euglenozoa) program sponsored by the National Science Foundation, USA (PI Gregory Buck: DEB-0830056), and by projects supported by CNPq, CAPES and USP to MMGT. ZEC is recipient of a PhD fellowship from SENACYT (Panamá). AGCM, RCF and JMPA are recipients of fellowships from Brazilian agencies CNPq, CAPES and FAPESP (grant \#2013/14622-3, São Paulo Research Foundation), respectively. We thank Marta Campaner for parasite cultures, and Carmen SA Takata and Tania E. Matsumoto for genes and genome sequencing (Illumina platform). We are grateful to Dr Carmenza Spadafora for valuable support given to this project in INDICASAT-AIP, Panama.

\section{Author details}

'Departamento de Parasitologia, Instituto de Ciências Biomédicas, Universidade de São Paulo, São Paulo, SP 05508-900, Brazil. ${ }^{2}$ Instituto de Investigaciones Científicas y Servicios de Alta Tecnología-AIP (INDICASAT-AIP), Ciudad del Saber, Clayon, Panamá. ${ }^{3}$ Department of Microbiology and Immunology, Virginia Commonwealth University, Virginia, USA. ${ }^{4}$ Département Infection et Epidemiologie, Institut Pasteur, Laboratoire des Processus Infectieux à Trypanosomatidés, Paris, France.

Received: 25 October 2014 Accepted: 25 March 2015

Published online: 12 April 2015

\section{References}

1. Lukes J, Skalicky T, Tyc J, Votypka J, Yurchenko V. Evolution of parasitism in kinetoplastid flagellates. Mol Biochem Parasitol. 2014;195:115-22.

2. Maslov DA, Votypka J, Yurchenko V, Lukes J. Diversity and phylogeny of insect trypanosomatids: all that is hidden shall be revealed. Trends Parasitol. 2013;29:43-52.

3. Hamilton PB, Gibson WC, Stevens JR. Patterns of co-evolution between trypanosomes and their hosts deduced from ribosomal RNA and protein-coding gene phylogenies. Mol Phylogenet Evol. 2007:44:15-25.

4. Vallejo GA, Guhl F, Schaub GA. Triatominae-Trypanosoma cruzi/T. rangeli: Vector-parasite interactions. Acta Trop. 2009;1 10:137-47.

5. Cavazzana Jr M, Marcili A, Lima L, da Silva FM, Junqueira AC, Veludo HH, et al. Phylogeographical, ecological and biological patterns shown by nuclear (ssrRNA and gGAPDH) and mitochondrial (Cyt b) genes of trypanosomes of the subgenus Schizotrypanum parasitic in Brazilian bats. Int J Parasitol. 2010;40:345-55.

6. Minoprio P. Parasite polyclonal activators: new targets for vaccination approaches? Int J Parasitol. 2001;31:588-91.

7. Buschiazzo A, Goytia M, Schaeffer F, Degrave W, Shepard W, Gregoire C, et al. Crystal structure, catalytic mechanism, and mitogenic properties of Trypanosoma cruzi proline racemase. Proc Natl Acad Sci U S A. 2006;103:1705-10.

8. Chamond N, Cosson A, Coatnoan N, Minoprio P. Proline racemases are conserved mitogens: characterization of a Trypanosoma vivax proline racemase. Mol Biochem Parasitol. 2009;165:170-9.

9. Chamond N, Goytia M, Coatnoan N, Barale JC, Cosson A, Degrave WM, et al. Trypanosoma cruzi proline racemases are involved in parasite differentiation and infectivity. Mol Microbiol. 2005;58:46-60.

10. Chamond N, Gregoire C, Coatnoan N, Rougeot C, Freitas-Junior LH, da Silveira JF, et al. Biochemical characterization of proline racemases from the human protozoan parasite Trypanosoma cruzi and definition of putative protein signatures. J Biol Chem. 2003;278:15484-94. 
11. Reina-San-Martin B, Degrave W, Rougeot C, Cosson A, Chamond N, Cordeiro-Da-Silva A, et al. A B-cell mitogen from a pathogenic trypanosome is a eukaryotic proline racemase. Nat Med. 2000;6:890-7.

12. Coatnoan N, Berneman A, Chamond N, Minoprio P. Proline racemases: insights into Trypanosoma cruzi peptides containing D-proline. Mem Inst Oswaldo Cruz. 2009;104 Suppl 1:295-300.

13. Radkov AD, Moe LA. Bacterial synthesis of D-amino acids. Appl Microbiol Biotechnol. 2014;98:5363-74.

14. Horcajo P, Pedro MA, Cava F. Peptidoglycan plasticity in bacteria: stress-induced peptidoglycan editing by noncanonical D-amino acids. Microb Drug Resist. 2012;18:306-13.

15. Cardinale GJ, Abeles RH. Purification and mechanism of action of proline racemase. Biochemistry. 1968;7:3970-8.

16. Goytia M, Chamond N, Cosson A, Coatnoan N, Hermant D, Berneman A, et al. Molecular and structural discrimination of proline racemase and hydroxyproline-2-epimerase from nosocomial and bacterial pathogens. PLoS One. 2007;2:e885.

17. Fitzpatrick DA, Logue ME, Butler G. Evidence of recent interkingdom horizontal gene transfer between bacteria and Candida parapsilosis. BMC Evol Biol. 2008;8:181

18. Visser WF, Verhoeven-Duif NM, de Koning TJ. Identification of a human trans-3-hydroxy-L-proline dehydratase, the first characterized member of a novel family of proline racemase-like enzymes. J Biol Chem 2012;287:21654-62.

19. Coutinho L, Ferreira MA, Cosson A, Batista MM, Batista Dda G, Minoprio P, et al. Inhibition of Trypanosoma cruzi proline racemase affects host-parasite interactions and the outcome of in vitro infection. Mem Inst Oswaldo Cruz. 2009;104:1055-62.

20. Zingales B, Miles MA, Moraes CB, Luquetti A, Guhl F, Schijman AG, et al. Drug discovery for Chagas disease should consider Trypanosoma cruzi strain diversity. Mem Inst Oswaldo Cruz. 2014;109:828-33.

21. Zingales B, Miles MA, Campbell DA, Tibayrenc M, Macedo AM, Teixeira MMG, et al. The revised Trypanosoma cruzi subspecific nomenclature: rationale, epidemiological relevance and research applications. Infect Genet Evol. 2012;12:240-53.

22. Spera JM, Herrmann CK, Roset MS, Comerci DJ, Ugalde JE. A Brucella virulence factor targets macrophages to trigger B-cell proliferation. J Biol Chem. 2013:288:20208-16.

23. Bryan MA, Norris KA. Genetic immunization converts the Trypanosoma cruzi B-Cell mitogen proline racemase to an effective immunogen. Infect Immun. 2010;78:810-22.

24. Martins RM, Covarrubias C, Rojas RG, Silber AM, Yoshida N. Use of L-proline and ATP production by Trypanosoma cruzi metacyclic forms as requirements for host cell invasion. Infect Immun. 2009;77:3023-32.

25. Saye M, Miranda MR, Di Girolamo F, De Los Milagros Camara M, Pereira CA Proline modulates the Trypanosoma cruzi resistance to reactive oxygen species and drugs through a novel D, L-proline transporter. PLoS One. 2014;9:e92028

26. Batista JS, Rodrigues CM, Garcia HA, Bezerra FS, Olinda RG, Teixeira MMG, et al. Association of Trypanosoma vivax in extracellular sites with central nervous system lesions and changes in cerebrospinal fluid in experimentally infected goats. Vet Res. 2011;42:63.

27. D'Archivio S, Cosson A, Medina M, Lang T, Minoprio P, Goyard S. Non-invasive in vivo study of the Trypanosoma vivax infectious process consolidates the brain commitment in late infections. PLoS Negl Trop Dis. 2013;7:e1976.

28. Rodrigues AC, Ortiz PA, Costa-Martins AG, Neves L, Garcia HA, Alves JM, et al. Congopain genes diverged to become specific to Savannah, Forest and Kilifi subgroups of Trypanosoma congolense, and are valuable for diagnosis, genotyping and phylogenetic inferences. Infect Genet Evol. 2014;23:20-31.

29. Opperdoes FR, Michels PA. Horizontal gene transfer in trypanosomatids. Trends Parasitol. 2007;23:470-6.

30. Keeling PJ, Palmer JD. Horizontal gene transfer in eukaryotic evolution. Nat Rev Genet. 2008:9:605-18.

31. Alsmark C, Foster PG, Sicheritz-Ponten T, Nakjang S, Martin Embley T, Hirt RP. Patterns of prokaryotic lateral gene transfers affecting parasitic microbial eukaryotes. Genome Biol. 2013;14:R19.

32. Alves JM, Klein CC, da Silva FM, Costa-Martins AG, Serrano MG, Buck GA, et al. Endosymbiosis in trypanosomatids: the genomic cooperation between bacterium and host in the synthesis of essential amino acids is heavily influenced by multiple horizontal gene transfers. BMC Evol Biol. 2013;13:190.

33. Alves JM, Serrano MG, Maia Da Silva F, Voegtly LJ, Matveyev AV, Teixeira MMG, et al. Genome evolution and phylogenomic analysis of Candidatus Kinetoplastibacterium, the betaproteobacterial endosymbionts of Strigomonas and Angomonas. Genome Biol Evol. 2013;5:338-50.

34. Klein CC, Alves JM, Serrano MG, Buck GA, Vasconcelos AT, Sagot MF, et al. Biosynthesis of vitamins and cofactors in bacterium-harbouring trypanosomatids depends on the symbiotic association as revealed by genomic analyses. PLoS One. 2013;8:e79786.

35. Hirt RP, Alsmark C, Embley TM. Lateral gene transfers and the origins of the eukaryote proteome: a view from microbial parasites. Curr Opin Microbiol. 2014;23:155-62.

36. Silva DC, Silva RC, Ferreira RC, Briones MR. Examining marginal sequence similarities between bacterial type III secretion system components and Trypanosoma cruzi surface proteins: horizontal gene transfer or convergent evolution? Front Genet. 2013;4:143.

37. Oliveira P, Lima FM, Cruz MC, Ferreira RC, Sanchez-Flores A, Cordero EM, et al. Trypanosoma cruzi: Genome characterization of phosphatidylinositol kinase gene family (PIK and PIK-related) and identification of a novel PIK gene. Infect Genet Evol. 2014;25:157-65.

38. Lima L, Silva FM, Neves L, Attias M, Takata CS, Campaner M, et al. Evolutionary insights from bat trypanosomes: morphological, developmental and phylogenetic evidence of a new species, Trypanosoma (Schizotrypanum) erneyi sp. nov., in African bats closely related to Trypanosoma (Schizotrypanum) cruzi and allied species. Protist. 2012;163:856-72.

39. Hamilton PB, Teixeira MMG, Stevens JR. The evolution of Trypanosoma cruzi: the 'bat seeding' hypothesis. Trends Parasitol. 2012;28:136-41.

40. Lima L, Espinosa-Alvarez O, Hamilton PB, Neves L, Takata CS, Campaner M, et al. Trypanosoma livingstonei: a new species from African bats supports the bat seeding hypothesis for the Trypanosoma cruzi clade. Parasit Vectors. 2013;6:221.

41. Ferreira RC, De Souza AA, Freitas RA, Campaner M, Takata CS, Barrett TV, et al. A phylogenetic lineage of closely related trypanosomes (Trypanosomatidae, Kinetoplastida) of anurans and sand flies (Psychodidae, Diptera) sharing the same ecotopes in brazilian amazonia. J Eukaryot Microbiol. 2008;55:427-35.

42. Viola LB, Attias M, Takata CS, Campaner M, de Souza W, Camargo EP, et al Phylogenetic analyses based on small subunit rRNA and glycosomal glyceraldehyde-3-phosphate dehydrogenase genes and ultrastructural characterization of two snake Trypanosomes: Trypanosoma serpentis n. sp. from Pseudoboa nigra and Trypanosoma cascavelli from Crotalus durissus terrificus. J Eukaryot Microbiol. 2009;56:594-602.

43. Fermino BR, Viola LB, Paiva F, Garcia HA, de Paula CD, Botero-Arias R, et al. The phylogeography of trypanosomes from South American alligatorids and African crocodilids is consistent with the geological history of South American river basins and the transoceanic dispersal of Crocodylus at the Miocene. Parasit Vectors. 2013;6:313.

44. Lima L, Ortiz PA, da Silva FM, Alves JM, Serrano MG, Cortez AP, et al. Repertoire, genealogy and genomic organization of cruzipain and homologous genes in Trypanosoma cruzi, T. cruzi-like and other trypanosome species. PLoS One. 2012;7:e38385.

45. Castresana J. Selection of conserved blocks from multiple alignments for their use in phylogenetic analysis. Mol Biol Evol. 2000;17:540-52.

46. Huson DH, Scornavacca C. Dendroscope 3: an interactive tool for rooted phylogenetic trees and networks. Syst Biol. 2012;61:1061-7.

47. Pond SL, Frost SD, Muse SV. HyPhy: hypothesis testing using phylogenies. Bioinformatics. 2005;21:676-9.

48. Kelly S, Ivens A, Manna PT, Gibson W, Field MC. A draft genome for the African crocodilian trypanosome Trypanosoma grayi. Sci Data. 2014;1:140024.

49. Flores-Lopez CA, Machado CA. Analyses of 32 loci clarify phylogenetic relationships among Trypanosoma cruzi lineages and support a single hybridization prior to human contact. PLoS Negl Trop Dis. 2011;5:e1272.

50. Marcili A, Lima L, Cavazzana M, Junqueira AC, Veludo HH, Maia Da Silva F, et al. A new genotype of Trypanosoma cruzi associated with bats evidenced by phylogenetic analyses using SSU rDNA, cytochrome b and Histone H2B genes and genotyping based on ITS1 rDNA. Parasitology. 2009;136:641-55.

51. Maia Da Silva F, Junqueira AC, Campaner M, Rodrigues AC, Crisante G, Ramirez LE, et al. Comparative phylogeography of Trypanosoma rangeli and 
Rhodnius (Hemiptera: Reduviidae) supports a long coexistence of parasite lineages and their sympatric vectors. Mol Ecol. 2007;16:3361-73.

52. Maia Da Silva F, Marcili A, Lima L, Cavazzana Jr M, Ortiz PA, Campaner M, et al. Trypanosoma rangeli isolates of bats from Central Brazil: genotyping and phylogenetic analysis enable description of a new lineage using spliced-leader gene sequences. Acta Trop. 2009;109:199-207.

53. Marcili A, Valente VC, Valente SA, Junqueira AC, da Silva FM, Pinto AY, et al. Trypanosoma cruzi in Brazilian Amazonia: Lineages TCl and TClla in wild primates, Rhodnius spp. and in humans with Chagas disease associated with oral transmission. Int J Parasitol. 2009;39:615-23.

54. Truc $P$, Büscher $P$, Cuny $G$, Gonzatti Ml, Jannin J, Joshi $P$, et al. Atypical human infections by animal trypanosomes. PLoS Negl Trop Dis. 2013;7:e2256.

55. Berneman A, Montout L, Goyard S, Chamond N, Cosson A, d'Archivio S, et al. Combined approaches for drug design points the way to novel proline racemase inhibitor candidates to fight Chagas' disease. PLoS One. 2013;8:e60955.

56. Duz ALC, Vieira PMA, Roatt BM, Aguiar-Soares RDO, Cardoso JMO, Oliveira FCB, et al. The TCl and TCll Trypanosoma cruzi experimental infections induce distinct immune responses and cardiac fibrosis in dogs. Mem Inst Oswaldo Cruz. 2014;109:1005-13.

57. Segovia M, Carrasco HJ, Martinez CE, Messenger LA, Nessi A, Londono JC, et al. Molecular epidemiologic source tracking of orally transmitted Chagas disease, Venezuela. Emerg Infect Dis. 2013;19:1098-101.

58. Teixeira MMG, da Silva FM, Marcili A, Umezawa ES, Shikanai-Yasuda MA, Cunha-Neto E, et al. Short communication: Trypanosoma cruzi lineage I in endomyocardial biopsy from a north-eastern Brazilian patient at end-stage chronic Chagasic cardiomyopathy. Trop Med Int Health. 2006;11:294-8.

59. Samudio F, Ortega-Barria E, Saldana A, Calzada J. Predominance of Trypanosoma cruzi I among Panamanian sylvatic isolates. Acta Trop. 2007;101:178-81.

60. Monteiro WM, Margioto Teston AP, Gruendling AP, dos Reis D, Gomes ML, de Araujo SM, et al. Trypanosoma cruzi I and IV stocks from Brazilian Amazon are divergent in terms of biological and medical properties in mice. PLoS Negl Trop Dis. 2013;7:e2069.

61. Ragone PG, Perez Brandan C, Padilla AM, Monje Rumi M, Lauthier JJ, Alberti D'Amato AM, et al. Biological behavior of different Trypanosoma cruzi isolates circulating in an endemic area for Chagas disease in the Gran Chaco region of Argentina. Acta Trop. 2012;123:196-201.

62. Llewellyn MS, Lewis MD, Acosta N, Yeo M, Carrasco HJ, Segovia M, et al. Trypanosoma cruzi llc: phylogenetic and phylogeographic insights from sequence and microsatellite analysis and potential impact on emergent Chagas disease. PLoS Negl Trop Dis. 2009;3:e510.

63. Guhl F, Auderheide A, Ramirez JD. From ancient to contemporary molecular eco-epidemiology of Chagas disease in the Americas. Int J Parasitol. 2014:44:605-12.

64. Ortiz PA, Maia Da Silva F, Cortez AP, Lima L, Campaner M, Pral EM, et al. Genes of cathepsin L-like proteases in Trypanosoma rangeli isolates: markers for diagnosis, genotyping and phylogenetic relationships. Acta Trop. 2009;112:249-59.

65. Urrea DA, Guhl F, Herrera CP, Falla A, Carranza JC, Cuba-Cuba C, et al. Sequence analysis of the spliced-leader intergenic region (SL-IR) and random amplified polymorphic DNA (RAPD) of Trypanosoma rangeli strains isolated from Rhodnius ecuadoriensis, R. colombiensis, R. pallescens and R. prolixus suggests a degree of co-evolution between parasites and vectors. Acta Trop. 2011;120:59-66.

66. Deane LM, Deane MP, Lourenco-de-Oliveira R. Are Asian monkeys the original mammalian hosts of Trypanosoma conorhini? Mem Inst Oswaldo Cruz. 1986;81:127-9.

67. Deane MP, Deane LM. Studies on the life cycle of Trypanosoma conorrhini. "In vitro" development and multiplication of the bloodstream trypanosomes. Rev Inst Med Trop Sao Paulo. 1961;3:149-60.

68. Beiko RG, Ragan MA. Detecting lateral genetic transfer: a phylogenetic approach. Methods Mol Biol. 2008;452:457-69.

69. Beiko RG, Ragan MA. Untangling hybrid phylogenetic signals: horizontal gene transfer and artifacts of phylogenetic reconstruction. Methods Mol Biol. 2009;532:241-56.

70. Wisecaver $\mathrm{JH}$, Hackett JD. The impact of automated filtering of BLAST-determined homologs in the phylogenetic detection of horizonta gene transfer from a transcriptome assembly. Mol Phylogenet Evol. 2014;71:184-92.
71. Strese A, Backlund A, Alsmark C. A recently transferred cluster of bacterial genes in Trichomonas vaginalis - lateral gene transfer and the fate of acquired genes. BMC Evol Biol. 2014;14:119.

72. Yutin N, Galperin MY. A genomic update on clostridial phylogeny: Gram-negative spore formers and other misplaced clostridia. Environ Microbiol Rep. 2013;15:2631-41.

73. Galen BT, Banach DB, Gitman MR, Trow TK. Meningoencephalitis due to Gemella haemolysans. J Med Microbiol. 2014;63:138-9.

74. Alves WCL, Gorayeb IS, Loureiro CB. Isolated bacteria from hematophagous Culicidae (Diptera:Nematocera) in Belém, Pará State, Brazil. Rev Pan-Amaz Saude. 2010;1:131-41.

\section{Submit your next manuscript to BioMed Central and take full advantage of:}

- Convenient online submission

- Thorough peer review

- No space constraints or color figure charges

- Immediate publication on acceptance

- Inclusion in PubMed, CAS, Scopus and Google Scholar

- Research which is freely available for redistribution 